\title{
Hydrometeor classification through statistical clustering of polarimetric radar measurements: a semi-supervised approach
}

\author{
Nikola Besic ${ }^{1,2}$, Jordi Figueras i Ventura ${ }^{2}$, Jacopo Grazioli ${ }^{1}$, Marco Gabella ${ }^{2}$, Urs Germann ${ }^{2}$, and Alexis Berne ${ }^{1}$ \\ ${ }^{1}$ Environmental Remote Sensing Laboratory (LTE), École Polytechnique Fédérale \\ de Lausanne (EPFL), Lausanne, Switzerland \\ ${ }^{2}$ Radar, Satellite and Nowcasting Department, MeteoSwiss, Locarno-Monti, Switzerland
}

Correspondence to: Nikola Besic (nikola.besic@epfl.ch)

Received: 29 March 2016 - Published in Atmos. Meas. Tech. Discuss.: 30 March 2016

Revised: 24 August 2016 - Accepted: 25 August 2016 - Published: 8 September 2016

\begin{abstract}
Polarimetric radar-based hydrometeor classification is the procedure of identifying different types of hydrometeors by exploiting polarimetric radar observations. The main drawback of the existing supervised classification methods, mostly based on fuzzy logic, is a significant dependency on a presumed electromagnetic behaviour of different hydrometeor types. Namely, the results of the classification largely rely upon the quality of scattering simulations. When it comes to the unsupervised approach, it lacks the constraints related to the hydrometeor microphysics. The idea of the proposed method is to compensate for these drawbacks by combining the two approaches in a way that microphysical hypotheses can, to a degree, adjust the content of the classes obtained statistically from the observations. This is done by means of an iterative approach, performed offline, which, in a statistical framework, examines clustered representative polarimetric observations by comparing them to the presumed polarimetric properties of each hydrometeor class. Aside from comparing, a routine alters the content of clusters by encouraging further statistical clustering in case of non-identification. By merging all identified clusters, the multi-dimensional polarimetric signatures of various hydrometeor types are obtained for each of the studied representative datasets, i.e. for each radar system of interest. These are depicted by sets of centroids which are then employed in operational labelling of different hydrometeors. The method has been applied on three C-band datasets, each acquired by different operational radar from the MeteoSwiss Rad4Alp network, as well as on two X-band datasets acquired by two research mobile radars. The results are discussed through a comparative analysis which includes a cor-
\end{abstract}

responding supervised and unsupervised approach, emphasising the operational potential of the proposed method.

\section{Introduction}

Radar-based hydrometeor classification, that is the proper identification of different types of hydrometeors from radar observations, is important for an improved understanding of atmospheric dynamics, an improved quantitative precipitation estimation (QPE), an improved verification and assimilation in numerical weather prediction models and operational nowcasting applications like aircraft or road safety (Bringi et al., 2007). The spread of polarimetry for weather radar has significantly changed the capability of radar systems to identify meteorological and non-meteorological echoes, as well as to identify different hydrometeor types under the radar umbrella (Bringi et al., 2007). The very first efforts to overcome the ambiguity arising from the overlap of measured reflectivity for different hydrometeors relied on a dual-polarisation parameter - the differential reflectivity (Seliga and Bringi, 1976; Hall et al., 1984). Since then, various methods, incorporating other Doppler dual-polarisation (called polarimetric hereafter) parameters, have been developed for the three frequency bands of major interest (S, C and X) (Bringi et al., 2007). Conceptually, all these methods can be categorised as supervised, unsupervised or semisupervised (Fig. 1).

Being by far the largest (Chandrasekar et al., 2013), the first category encompasses mostly approaches based on Boolean logic decision tree, Bayesian theory and the most 


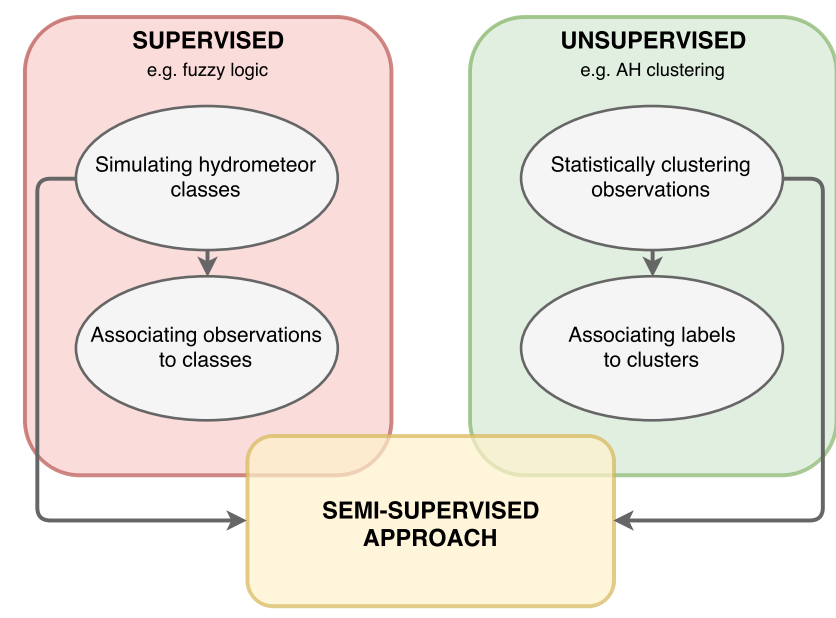

Figure 1. Schematic generalisation of hydrometeor classification methods.

intuitive ones, based on fuzzy logic. The common ground for these seemingly different approaches is a necessity for a very reliable set of polarimetric signatures (Straka et al., 2000). These are obtained either by means of simulations (e.g. Dolan and Rutledge, 2009) or by additionally involving some empirical knowledge (e.g. Al-Sakka et al., 2013).

Boolean logic decision tree methods are part of the earliest efforts to exploit radar polarimetry for the purpose of hydrometeor classification (Straka and Zrnic, 1993; El-Magd et al., 2000). However, assuming mutual exclusivity of polarimetric parameters for different hydrometeor types, these methods could not thoroughly exploit the potential of polarimetric measurements.

Fuzzy logic has been considered to be the best way to make use of polarimetric signatures known a priori in distinguishing between different hydrometeors (Vivekanandan et al., 1999). Namely, inference combining matching scores of different parameters with overlapping membership functions precisely overcomes the mutual exclusivity limits of the Boolean logic decision tree and makes methods less susceptible to the potential presence of noise. Among the number of methods developed for S, C and X bands we can distinguish between those using reflectivity at horizontal polarisation $\left(Z_{\mathrm{H}}\right)$, differential reflectivity $\left(Z_{\mathrm{DR}}\right)$, specific differential phase shift $\left(K_{\mathrm{dp}}\right)$ and correlation coefficient $\left(\rho_{\mathrm{hv}}\right)$ (e.g. Dolan et al., 2013) and those using linear depolarisation ratio (LDR) (e.g. Straka et al., 2000). Also, we can discriminate between methods using temperature as an external parameter (e.g. Zrnic et al., 2001), rather than relative altitude with respect to the $0{ }^{\circ} \mathrm{C}$ isotherm (e.g. Lim et al., 2005), as well as between those using two-, or more, dimensional membership functions (e.g. Marzano et al., 2007), rather than one-dimensional ones (e.g. Liu and Chandrasekar, 2000).

As the most widespread approach in hydrometeor classification, fuzzy-logic classification methods have been subject of several validation campaigns. One of the most ex- tensive, the Joint Polarization Experiment (Ryzhkov et al., 2005), demonstrated improved hail detection capabilities using ground measurements with hail-intercept vehicles. A Bayesian approach proposed by Marzano et al. (2010) is another representative supervised approach, in which each simulated class is characterized by its centre and covariance matrix. The labelling of the observations is done by means of Bayesian inference (the maximum a posteriori rule).

The most obvious limitation of this dominant class of methods is the significant conditionality of the classification decision to the quality of the supposedly known a priori polarimetric signatures.

A different approach, based on the unsupervised concept and pioneered by Grazioli et al. (2015), tends to avoid using a priori known or presumed polarimetric signatures. The focus is rather on exploiting the radar observations with the aim of clustering a set of diverse polarimetric measurements into distinct clusters which are to be labelled as different hydrometeor types. In the mentioned method, the separation is achieved through agglomerative hierarchical clustering (AHC) and by simultaneously introducing a spatial texture information. The labelling of obtained clusters is done once manually by taking into account both radar and nonradar information.

The first notion of the semi-supervised method is found in Liu and Chandrasekar (2000), in which employing neural networks in updating the membership functions reduces classification algorithm to a neuro-fuzzy system. However, the most representative for this category would be a regionbased method proposed in Bechini and Chandrasekar (2015), the emphasis of which is on improving the output of fuzzylogic schema by applying clustering with a significant spatial (regional) constraint, which introduces an important robustness with respect to the inevitable noise in the measurements.

As was the case with the introduced unsupervised approach, the idea behind the semi-supervised approach we propose here is to avoid heavily relying on the presumed polarimetric properties of hydrometeors, though not entirely. Namely, the intentions were (i) to allow for the "glimpse" of the presumed hydrometeor microphysical properties through a constrained clustering and simultaneously; (ii) to automatise labelling of the obtained clusters (influence of the supervised concept); (iii) to make the classification decision criteria conform to the data specificities, particularly potential imperfections of the radar measurements (influence of the unsupervised concept); and (iv) to ensure the operational potential of the method by keeping the implementation simple enough for real-time operation.

This is achieved by involving, in a computationally efficient bin-based approach, two classical data processing tools: $k$-medoids clustering and Kolmogorov-Smirnov (KS) test. On one hand, the "glimpse" of the presumed microphysics comes through the appropriately modified state-of-the-art assumptions (Dolan and Rutledge, 2009; Dolan et al., 2013). On the other hand, the influence of the technical specifici- 
ties of radar is taken into account by comparatively working on datasets acquired by three MeteoSwiss Rad4Alp (Germann et al., 2015) C-band operational radars (Albis, Monte Lema and Plaine Morte) and two X-band mobile radars (MXPol, belonging to École Polytechnique Fédérale de Lausanne (EPFL), and DX50, operated by MeteoSwiss). As a result, we obtain for each of the considered radars a set of centroids in a multi-dimensional space, formed by four polarimetric parameters and a liquid/melting/ice phase indicator. These are later used to classify observed precipitation by simply applying an Euclidean distance criterion, which makes the method very suitable for operational use. Qualitative and quantitative performance tests are performed through comparison with the appropriate supervised and unsupervised routines, as well as through comparison with hydrometeor types observed at ground level with a two-dimensional video disdrometer (2DVD), and by involving external information, such as hail operational product and rain-gauge measurements.

The article is organised as follows: in Sect. 2 we introduce the employed statistical methods. Section 3 contains a detailed description of the proposed method, along with some auxiliary analyses. In Sect. 4 we illustrate some results of the classification applied to $\mathrm{C}$ - and X-band datasets and simultaneously analyse them through appropriate comparisons with independent measurements. Finally, Sect. 5 concludes the article through a discussion and provides some perspectives.

\section{Background on employed statistical methods}

The proposed semi-supervised algorithm mainly relies on two statistical tools, elaborated in the following subsections: the unsupervised $k$-medoids clustering and the KS statistical test. These two methods serve as a sort of link between the polarimetric radar measurements and the hydrometeor scattering hypotheses.

\subsection{Unsupervised clustering}

As it would be the case with the $k$-means (Lloyd., 1982), the employed $k$-medoids algorithm (Kaufman and Rousseeuw, 2009 ) is used to partition the multivariate observation vectors $\left(\boldsymbol{x}_{1}, \boldsymbol{x}_{2}, \ldots \boldsymbol{x}_{n}\right)$ into $k$ subsets or clusters $\left(S_{1}, S_{2}, \ldots S_{k}\right)$ in such a way that the subsets minimise $D$, the sum of distances between the observations and the centroids of subset $\boldsymbol{\mu}_{i}$ :

$D=\sum_{i=1}^{k} \sum_{\boldsymbol{x} \in S_{i}} d_{i}\left(\boldsymbol{x}-\boldsymbol{\mu}_{i}\right)$

The distance $d$ can vary from squared Euclidean norm $\|\cdot\|_{2}^{2}$ default $k$-means distance, $\ell_{1}$ norm (introduced by (Kaufman and Rousseeuw, 1987), in the original $k$-medoids algorithm), to the standardised Euclidean distance:

$d_{i}=\left\|\frac{\boldsymbol{x}-\boldsymbol{\mu}_{i}}{\boldsymbol{\sigma}_{S_{i}}}\right\|_{2}$, which is normalised with respect to the standard deviation of the subset $\left(\sigma_{S_{i}}\right)$ that we have adopted for our approach. It is an iterative algorithm, where centroids are recalculated after each iteration, during which the composition of the subsets changes. Once the composition becomes stationary, the algorithm has converged. Unlike the case for the $k$-means, where a centroid does not necessarily belong to the dataset, the centroid of a subset in the $k$-medoids algorithm, named medoid, is always a member of a set. This makes $k$-medoid more robust to the presence of outlier data, particularly when partitioning smaller sets of observations. The implementation of the method depends on the size of the observations sample, following criteria of the default MathWorks (2015) version.

- For small samples (up to 3000 observations), we employ the partitioning around medoids (PAM) algorithm (Kaufman and Rousseeuw, 2009). This procedure assumes minimising $D$ by swapping between medoids and non-medoids.

- For large samples (from 3000 to 10000 observations), an algorithm proposed in Park and Jun (2009) is used. The minimisation of $D$ is achieved as in the case of $k$ means, by choosing the closest medoid to the hypothetical corresponding $k$-means centroid.

- For very large samples (more than 10000 observations), only a random selection of cluster's samples is considered in recalculating medoids.

As foreboded in the introduction, the vector $\boldsymbol{x}$ has five dimensions in our case: four polarimetric parameters and a liquid/melting/ice phase sigmoidal indicator. Different distributions are characterized with different kurtosis (e.g. $Z_{\mathrm{H}}$ usually has far more negative kurtosis than $K_{\mathrm{dp}}$ ) and therefore the need to standardise (normalise) the Euclidean distance by dividing it by the standard deviation of the considered variable.

\subsection{Kolmogorov-Smirnov test}

The two-sample KS test is a non-parametric hypothesis test which tells us whether two samples can be characterized with the same probability distribution, whereas the one-sample version determines whether the sample is distributed according to particular distribution (Kolmogorov, 1933; Smirnov, 1948).

The test itself is based on the comparison between empirical cumulative distribution functions $(F)$ of two samples, the test statistic $D^{\mathrm{KS}}$ being the supremum of the set of their distances:

$D^{\mathrm{KS}}=\sup _{x_{j}}\left(\left|F_{1}\left(x_{j}\right)-F_{2}\left(x_{j}\right)\right|\right), x_{j} \in \boldsymbol{x}$,

with the absolute value making the test two-tailed. The decision to accept the $H_{0}$ hypothesis, which assumes that two 
samples are being derived from the same probability distribution, is made either by comparing the test statistic with the critical value or by comparing the $p$ value with the test significance $\alpha$ (type I error). Through the dependence of critical value and $p$ value on the number of samples, related to the test power $1-\beta$, the test decision depends as well on $\beta$ (type II error). A type I error is the false rejection of a true $H_{0}$ hypothesis, while a type II error is the failure to reject a false $H_{0}$ hypothesis.

In our case, the two samples are the values of the observed parameter $x_{j}$ (one of the five considered parameters) and the expected values of the same parameter (issued from the employed membership functions), as will be elaborated in the following section. The decision is based on comparing the value of the test statistic with the critical value as determined by Pearson and Hartley (1972).

\section{Algorithm}

The algorithm contains the "offline" part, which does not run in real time, and the "online" part, which runs operationally, as part of the processing chain of a radar network. The former is the core of the presented semi-supervised method. In this part the classification is configured through the preparation of representative dataset and the derivation of centroids. The latter part assumes the labelling of sample volumes as well as the estimation of the classification uncertainty.

\section{1 "Offline" algorithm}

The process starts with the selection of representative observations, aiming to get a dataset which contains all hydrometeor types we should potentially be able to detect and identify: crystals (CR), aggregates (AG), light rain (LR), rain $(\mathrm{RN})$, rimed ice particles (RP), vertically aligned ice (VI), wet snow (WS), ice hail and high-density graupel (IH) and melting hail $(\mathrm{MH})$. This choice of classes, inspired by Dolan and Rutledge (2009) and Dolan et al. (2013), and finally concluded by taking into account the operational preferences, represents a conventional set of hydrometeor types, with the exception of a strongly emphasized hail, which is present in two classes. At X band, with the MXPol radar, we did not manage to observe any vertically aligned ice while collecting representative observations and, thus, we had to omit the VI class. Nevertheless, this selection of classes is not mandatory, because the proposed approach can be used with any set of hydrometeor classes; i.e. new classes can be added quite easily.

\subsubsection{Data preparation}

At $\mathrm{C}$ band, representative observations are selected by carefully sampling 8 days of radar measurements for Albis and Monte Lema radars and 4 days for Plaine Morte radar, involving several stratiform and convective precipitation events (from all four seasons). The reason behind the smaller initial set for Plaine Morte radar (4 days) is the lower regional frequency of hail storms and our desire to keep the proportions of different hydrometeors similar for all radars. All three considered operational radars have the same scanning pattern covering the entire azimuth revolution with 20 elevations (plan position indicators (PPIs) from -0.2 to $40^{\circ}$ ) in $5 \mathrm{~min}$, resulting in 288 full scans per day. Clutter contaminated pixels, as identified by the slightly adjusted (emphasising the role of polarimetry) operational clutter removal routine (Germann and Joss, 2004), as well as the pixels below the noise level threshold, are removed. However, the sampling is restricted to the elevations from 3.5 to $11^{\circ}$, as well as to the range between 3 and $40 \mathrm{~km}$. The lower elevation boundary was chosen to avoid any potential residual clutter, while the upper one, combined with the selected range, restricts the considered altitude below $7.5 \mathrm{~km}$, sufficient to sample all types of precipitating hydrometeors.

Selection itself is a sort of constrained random sampling. As an effort to encourage the diversity of present hydrometeor types, we aim to obtain the distributions of parameters (particularly $Z_{\mathrm{H}}$ ), as platykurtic as possible, in the following ranges:

$$
\begin{aligned}
& \text { 1. } Z_{\mathrm{H}}:-10-60 \mathrm{dBZ}, \\
& \text { 2. } Z_{\mathrm{DR}}:-1.5-5 \mathrm{~dB}, \\
& \text { 3. } K_{\mathrm{dp}}:-0.5-5 \mathrm{deg} \mathrm{km} \mathrm{km}^{-1} \text {, } \\
& \text { 4. } \rho_{\mathrm{hv}}: 0.7-1, \\
& \text { 5. Ind: }-1-1 .
\end{aligned}
$$

The values of selected polarimetric parameters can be associated with different microphysical properties of the particles: $Z_{\mathrm{H}}$ indicates concentration, size and density, $Z_{\mathrm{DR}}$ indicates shape, orientation and density, $K_{\text {dp }}$ indicates concentration and shape and $\rho_{\mathrm{hv}}$ indicates homogeneity. Therefore, information they provide is to a degree complementary and should lead to the inference of a particle type. However, given that radar measurements are in fact estimates of these parameters, these values are characterized with a certain level of uncertainty and are the presumed polarimetric signatures of different particles. The proposed method is actually designed to adapt to these uncertainties.

The fifth parameter is introduced to better distinguish classes in liquid and ice phase that have similar polarimetric signatures, but without directly introducing the information about temperature. It is not directly observed by radar but can be deduced from radar measurements in stratiform precipitation by identifying the melting layer. It is finally a quasi-balanced ternary system indicating liquid, melting and ice phase, obtained by applying a sigmoid transform in order to decrease its influence on discrimination between different hydrometeor types inside liquid or ice phase:

$$
\text { Ind }=\frac{2}{1+e^{-b \Delta H}}-1,
$$




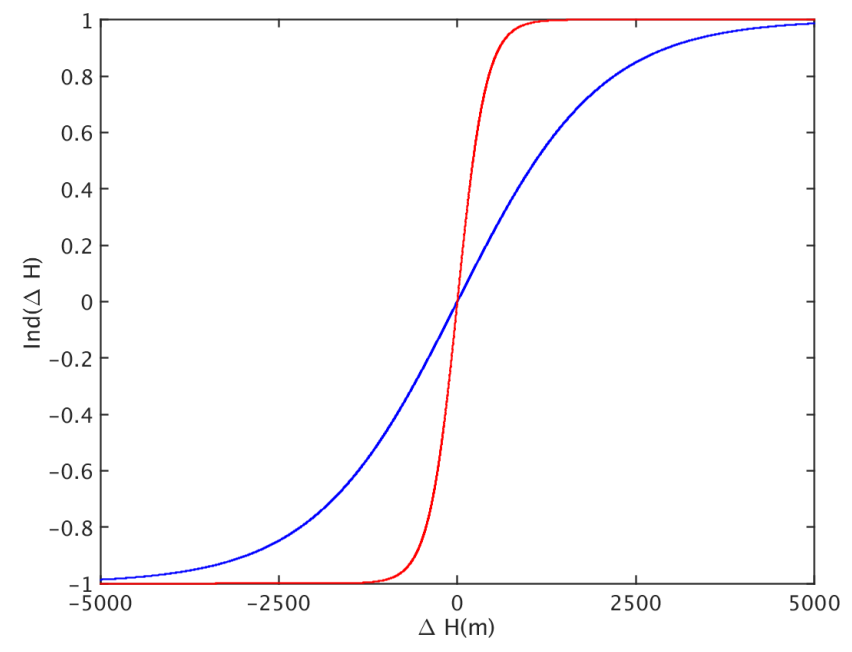

Figure 2. Sigmoid transformation: centroid derivation (blue) and pixels assignment (red).

where $\Delta H$ is a relative altitude with respect to the $0^{\circ}$ isotherm, and a slope parameter $b$ is either very low (blue curve in Fig. 2) or very large (red curve). The former one is applied in centroid derivation, while the latter is used in the assignment. The rationale behind the less steep slope applied on the representative observations is preserving a sort of continuity, for the purpose of the coherent statistical testing of five continuous distributions. Namely, the radar polarimetric variables are continuous, in both the centroid derivation and the assignment part. Therefore, given that we combine the scores of five-dimensional KS tests (as it will be elaborated in the following subsection), we decided to soften the "blue" transformation in order to make this parameter also continuous in the part where the KS test is used. This softer transformation still, however, limit the influence of $\Delta H$ on the classification.

The set of representative observations at $\mathrm{X}$ band is the one used in illustrating the unsupervised approach proposed in Grazioli et al. (2015). Given the transportability of the employed radar, these datasets were collected at two different locations, in two different climatic regions (Davos in Switzerland and Ardèche in France), at elevation angles ranging from 3.5 to $10^{\circ}$.

The sizes of the derived representative datasets, along with the most relevant information about the considered radars, are given in Table 1.

The information concerning the altitude of the $0^{\circ} \mathrm{C}$ isotherm has been collected from the numerical weather prediction model COSMO (Baldauf et al., 2011) by relying on the $0^{\circ} \mathrm{C}$ isotherm product or by applying standard atmosphere lapse rate in the troposphere $\left(6.4^{\circ} \mathrm{C} \mathrm{km}^{-1}\right)$ on the temperature profiles. The exceptions are stratiform events observed with the X-band radars, for which the melting layer is detected using a polarimetric radar-based method
(Wolfensberger et al., 2015). The idea is to do the same in the case of stratiform events observed with the C-band radar.

The concise description of the processing of the employed datasets is provided in Appendix B.

\subsubsection{Centroids derivation}

The method itself is conceived as an iteration inside an iteration. In this section we intend to provide a detailed description, starting from the "internal loop" and going towards the external one, the latter resulting in a final set of centroids for the considered radar.

As can be seen in Fig. 3, the "internal loop" is the very core of the proposed method. It starts with an initial, entirely unsupervised clustering of the representative dataset. This is done by means of the $k$-medoids clustering algorithm, which divides the set of representative observations into $N$ initial, distant sets by using the standardised Euclidean distance as a criterion. The number of initial sets is set to nine, which corresponds to the number of hydrometeor classes we eventually seek (see introduction to Sect. 3.1). A different number of initial sets does not alter the convergence of the algorithm.

Further on, each of these clusters is compared to the reference observations (identification), issued from onedimensional membership functions given in Appendix A. These observations are issued independently for each considered hydrometeor class by means of the inverse sampling method. Inverse sampling method generates the reference values by using the inverse version of the normalised cumulative sum of the reference parametric distribution. We supply it with a sequence of random uniformly distributed numbers (between 0 and 1 ) and obtain at the output the set of observations whose distribution is determined by the parameters of the membership function. The basis of these membership functions is adopted from the state of the art (Dolan et al., 2013; Dolan and Rutledge, 2009), and further appropriately modified and enriched by means of scattering simulations based on single- and double-layer T-matrix method (Mishchenko et al., 1996), as elaborated in Appendix A. The comparison itself is performed using the KS test (see Sect. 2.2) by comparing separately each of the five considered parameters. Then, the five obtained test statistics are combined using a weighted arithmetic sum:

$D^{\mathrm{KS}}=\frac{w_{1} D_{Z_{\mathrm{H}}}^{\mathrm{KS}}+w_{2} D_{\mathrm{Z}_{\mathrm{DR}}}^{\mathrm{KS}}+w_{3} D_{K_{\mathrm{dp}}}^{\mathrm{KS}}+w_{4} D_{\rho_{\mathrm{hv}}}^{\mathrm{KS}}+w_{5} D_{\mathrm{Ind}}^{\mathrm{KS}}}{\sum_{i=1}^{5} w_{i}}$,

with $w_{i}=1$ for $i=1 \ldots 4$ and $w_{5} \leq 0.75$, the latter being part of the endeavour to decrease the impact of non-radar variables. The resultant test statistic is finally compared with the critical value defined by a chosen test significance $(\alpha)$ and a number of samples $(\beta)$, following Pearson and Hartley (1972).

Clusters which satisfy the $H_{0}$ hypothesis (distribution similar enough to one of the reference classes) exit the iteration 
Table 1. Basic information about the four observations datasets.

\begin{tabular}{lllll}
\hline Radar & $\begin{array}{l}\text { Band: frequency } \\
(\mathrm{GHz})\end{array}$ & $\begin{array}{l}\text { Range resolution } \\
(\mathrm{m})\end{array}$ & $\begin{array}{l}\text { Altitude } \\
(\mathrm{m})\end{array}$ & $\begin{array}{l}\text { Obs. dataset size } \\
\text { (pixels) }\end{array}$ \\
\hline Albis & C: 5.45 & 83 & 938 & 235359 \\
Monte Lema & C: 5.46 & 83 & 1626 & 217598 \\
Plaine Morte & C: 5.47 & 83 & 2937 & 209404 \\
MXPol & X: 9.41 & 75 & 2133 and 605 & 189675 \\
DX50 & X: 9.51 & 75 & 450 & 216675 \\
\hline
\end{tabular}

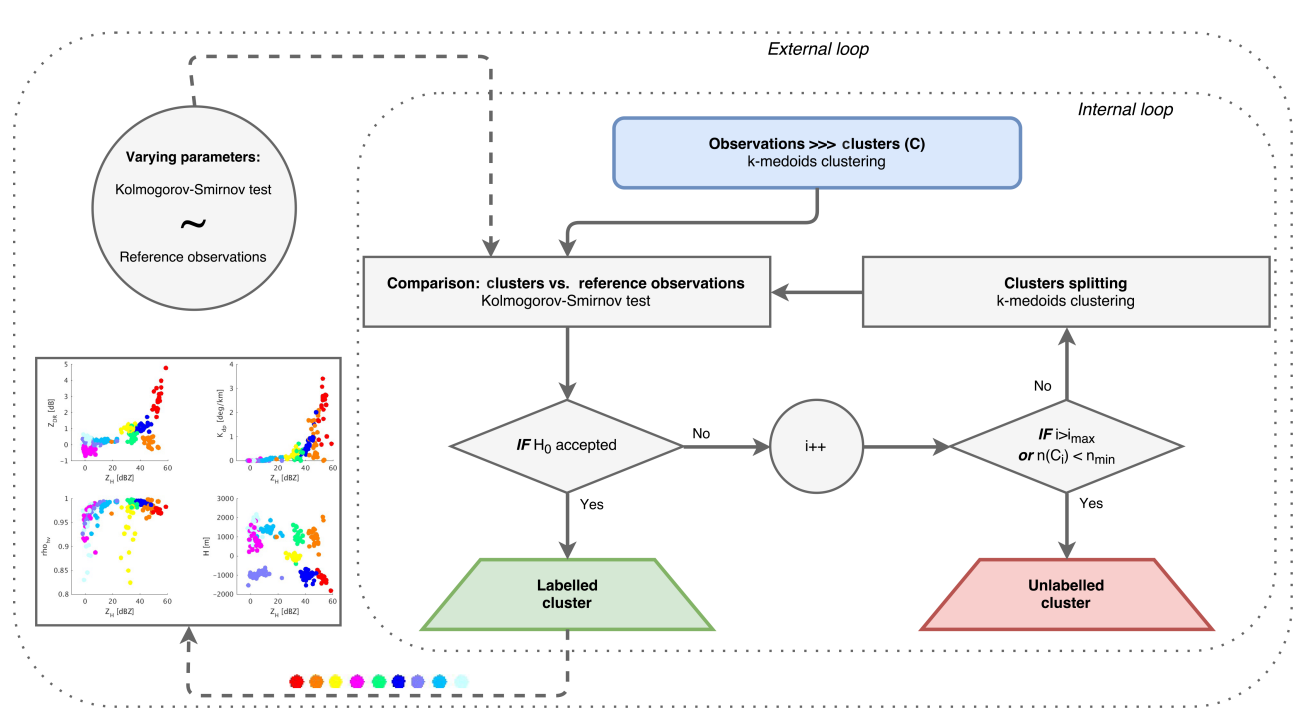

Figure 3. The schematic representation of the centroids derivation algorithm.

as labelled observations, while the rest proceeds to the additional clustering, this time divided into two sets. This clustering procedure is identical to the initial one, entirely unsupervised. The potential benefit of including the information about dissimilarity, arising from the KS test, which is the value of $x_{j}$ for which we have the maximal distance $D^{\mathrm{KS}}$ (Eq. 3), was investigated but it turned out that a constrained clustering would in fact not be beneficial. Namely, both the identification rate and the credibility of the obtained classes were in favour of an unconstrained clustering.

The obtained new sets are again undergoing identification, separately. The loop for a cluster which fails to be identified ends when the number of iterations exceeds $i_{\max }$, which is empirically determined to at least 10 , or when the size of the cluster falls below $n_{\min }$, which is identical to the number of samples, a parameter that varies in the external loop. All the labelled clusters are merged, according to the assigned label, into nine classes, which are characterized by a set of nine centroids in the five-dimensional space. However, unlabelled clusters are assumed to be mixtures of hydrometeor types that have more equal contributions to the polarimetric variables and therefore are not further analysed in this phase of our research. Their proportion is minimised by considering a relatively close range (up to $40 \mathrm{~km}$ ) in selecting representative observations.

As it was the case with the clustering method, the selected comparison method has been also investigated, by implementing in parallel Student's $t$ test (Snedecor and Cochran, 1989) and Wilcoxon's rank sum test (Gibbons and Chakraborti, 2011). The former is focused on the equality of mean values under the assumption of equal variances of normally distributed samples, while the latter examines equality of medians without any additional constraints. The identification with both of these alternative tests is a bit faster with respect to the KS test (Fig. 4), which can be explained by the fact that the KS test relies on the entire probability density function (all moments), unlike the studied alternatives which consider only first-order statistics. However, the composition of the obtained classes does not vary significantly, leading us to the decision to keep the KS test due to less restraining assumptions.

In the external loop, the previously described algorithm is run up to 30 times, with random variations of the $\mathrm{KS}$ test parameters and reference observations. Namely, in order to keep the $\alpha \beta$ product quasi-constant, we only slightly vary the number of samples $S \in[30,35,40]$ and therefore the $\beta$ parameter, whereas we keep the low value of the test sig- 


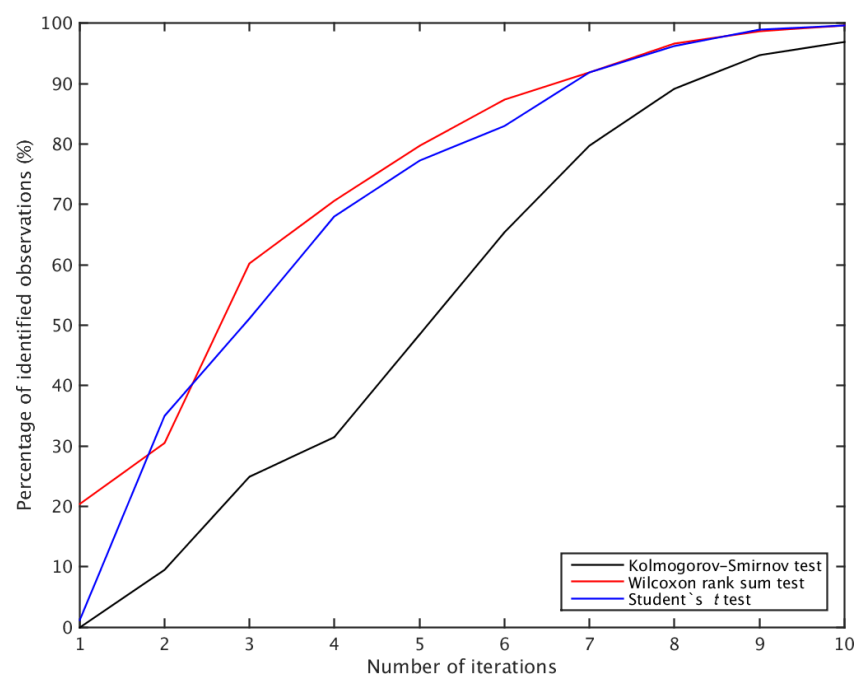

Figure 4. Comparison of clusters identification rates depending on an employed statistical test.

nificance $\alpha=0.01$. In contrast, the parameters of the presumed distributions of polarimetric variables (reference observations), $m, a, b$ as well as $x_{1}, x_{2}, x_{3}$ and $x_{4}$, are basically uniformly distributed in a $5 \%$ range around the values given in Appendix A.

The obtained classes from each of these 30 external iterations are firstly used to estimate multi-dimensional probability density functions (PDFs) for each of the detected hydrometeor types. This is done by means of a kernel density estimation (KDE) (Ihler and Mandel, 2003; Parzen, 1962). The resulting polarimetric descriptors have the potential to be further on used as a non-parametric membership function in a fuzzy-logic classification algorithm (Wen et al., 2015, 2016). Though, in this paper their role is restricted to the qualitative description of the obtained classes. A comparative illustration of the PDFs obtained by MXPol and DX50 radars (Fig. 5) shows a fairly similar two-dimensional distributions except in what concerns $\rho_{\mathrm{hv}}$, where the obtained PDFs and consequently the representative centroid reflect the systematic underestimation of this parameter by the DX50 radar. This sort of adaptability is the basic idea of the proposed classification method.

The proposed classification requires a final set of centroids, composed out of medians of centroids obtained in the external loop (Fig. 6). However, before defining a final centroid for a given hydrometeor class, we check for the dispersion of the 30 centroids obtained in the considered fivedimensional space. This is done by calculating the interquartile coefficient of dispersion, ranging from 0 to 1 , and conceived as

$$
c_{d}^{j, i}=\frac{Q_{75}^{j, i}-Q_{25}^{j, i}}{Q_{75}^{j, i}+Q_{25}^{j, i}},
$$

with $j$ standing for a polarimetric parameter and $i$ for a hydrometeor class. If the overall value of the coefficient (average over all five parameters), inversely proportional to the share of a given hydrometeor class in the representative set of observations, exceeds the empirically determined threshold (0.5), then the corresponding class is not being considered.

An important remark is that the increase of the variation range of reference parameters in the external loop, from 5 to $20 \%$ around their initial values, results in a very small change in the positions of centroids (e.g. average $\Delta Z_{\mathrm{DR}}=$ $0.0924 \mathrm{~dB}$ ) and a negligible change in their dispersion (average $\Delta c=0.0647$ ). This clearly indicates a limited sensitivity of the semi-supervised method on the employed reference assumptions.

As it can be deduced from Fig. 6, the differences between centroids characterising different $\mathrm{C}$-band radars do not appear to be too significant. Consequently, they do not alter considerably the outcome of the classification, which is demonstrated in Fig. 7, where Monte Lema centroids are applied on Albis and Plaine Morte radars. However, as we can observe in the latter figure, misclassification is still possible (e.g. AG vs. RP or WS vs. RN). Therefore, it appears that the idea of classification criteria being adapted to the particularities of a radar is relevant. This is especially justified in case of the $\rho_{\mathrm{hv}}$ parameter, which is sensitive to the quality of the antenna system of each operational radar.

The comparison with the centroids derived from unprocessed data (before attenuation and noise corrections) illustrates the rather significant influence the processing can have on the position of centroids. Despite this, the matching analysis between the classification performed with the "uncorrected" centroids on unprocessed dataset and the one performed with the "corrected" centroids on the same dataset after processing indicates some skills with respect to the data quality (65.3\% of observations on the normalised matching matrix diagonal).

\section{2 "Online" algorithm}

Once we have obtained the set of centroids characterising a particular radar, the operational implementation of hydrometeor classification comes down to the calculation of Euclidean distances in a five-dimensional space, formed by four parametric radar parameters and one external parameter (Ind). The configuration file containing centroids is supposed to be updated at regular intervals of several months in order to account for potentially occurring systematic errors in radar measurements, with the prospective of making this operation continuous.

\subsubsection{Pixels assignment}

Given the skewness and the leptokurticity characterising distributions of $K_{\mathrm{dp}}$ and $\rho_{\mathrm{hv}}$, these parameters are transformed into $K_{\mathrm{dp}}^{\prime}$ and $\rho_{\mathrm{hv}}^{\prime}$ by respectively applying the following 


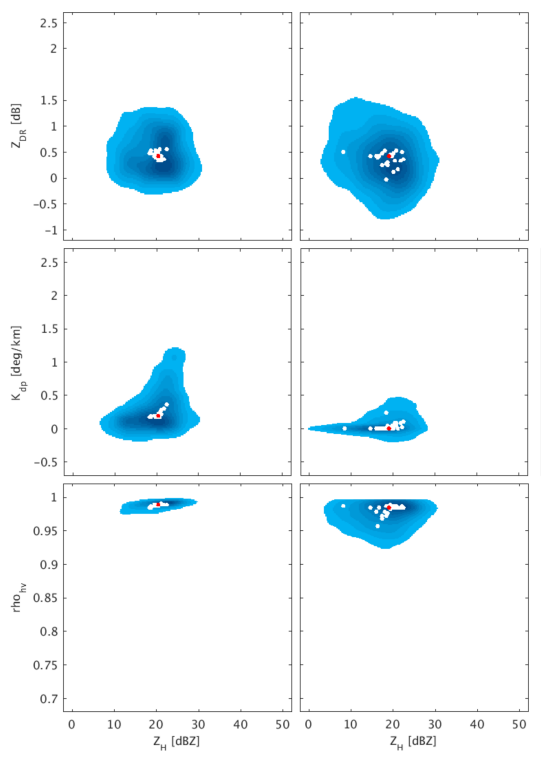

(a1)

(a2)

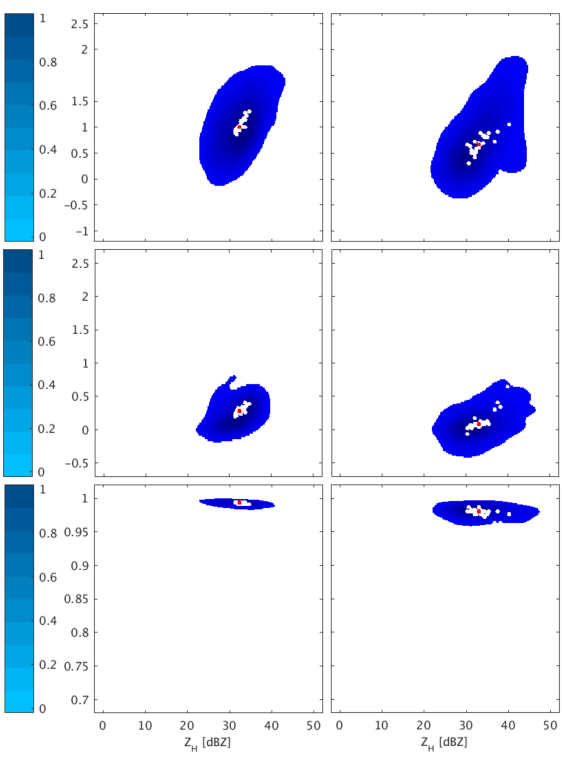

(b1)

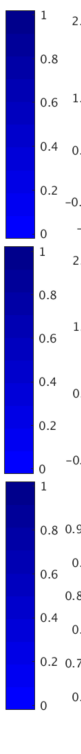

(b2)

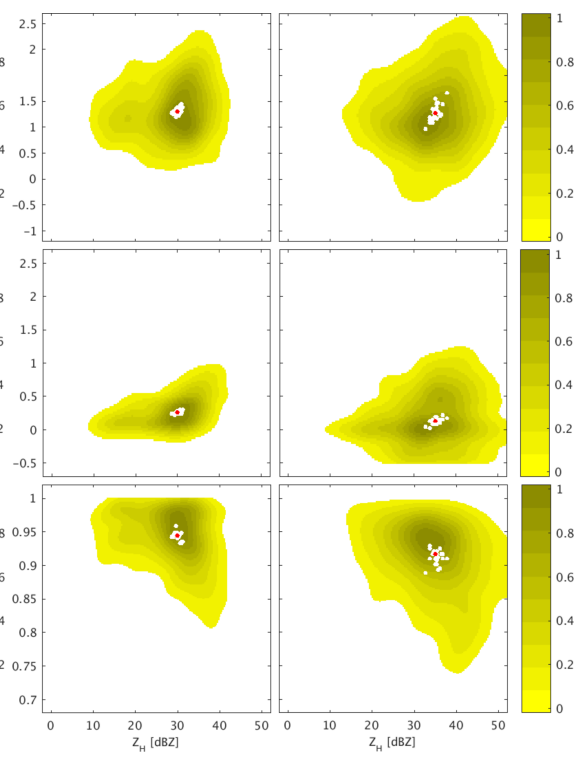

(c1)

(c2)

Figure 5. Examples of PDFs estimated by means of kernel density estimation (KDE) along with the centroids from all iterations (white) and final centroids (red), for MXPol (1) and DX50 (2) datasets: (a) AG, (b) RN, (c) WS.
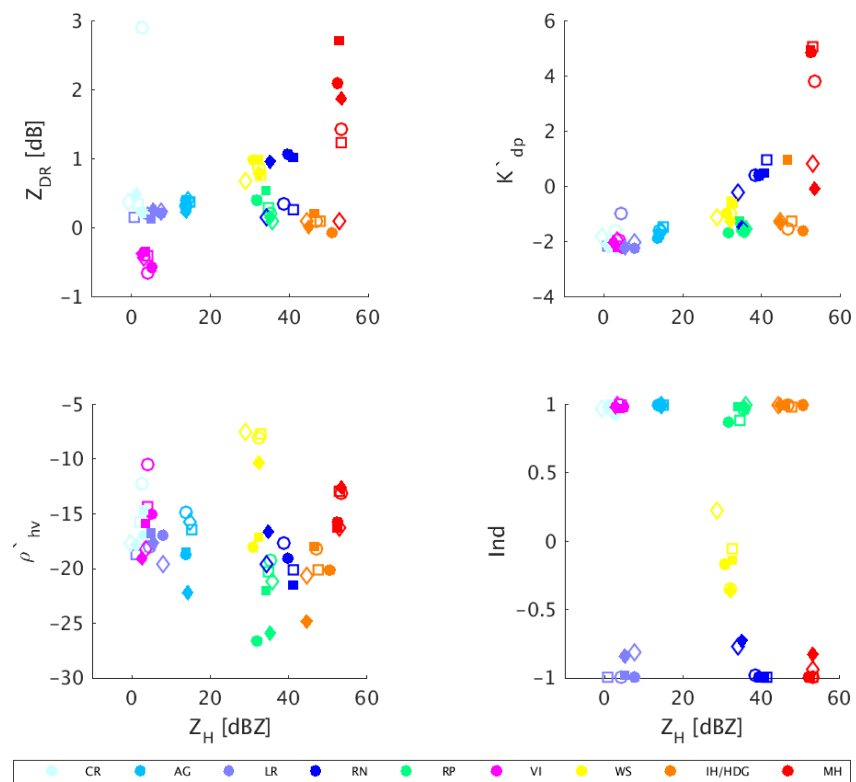

Figure 6. Centroids for Albis (o), Monte Lema $(\square)$ and Plaine Morte $(\diamond)$ radars: before (empty) and after (filled) attenuation and noise corrections. Classes: CR is crystals, AG is aggregates, LR is light rain, $\mathrm{RN}$ is rain, $\mathrm{RP}$ is rimed ice particles, VI is vertically aligned ice, WS is wet snow, IH/HDG is ice hail and high density graupel and $\mathrm{MH}$ is melting hail. $K_{\mathrm{dp}}^{\prime}$ and $\rho_{\mathrm{hv}}^{\prime}$ are defined in Eqs. (7) and (8) respectively.

transformations:

$K_{\mathrm{dp}}^{\prime}=10 \log \left(K_{\mathrm{dp}}+0.6\right)$,

$\rho_{\mathrm{hv}}^{\prime}=10 \log \left(1-\rho_{\mathrm{hv}}\right)$, with $K_{\mathrm{dp}}$ values being shifted for 0.6 to take into account $K_{\text {dp }}<0$.

In order to equalise their magnitudes, before proceeding to the pixel labelling, all the data, including the centroids, are scaled to the $[-1,1]$ range:

$x_{\text {out }}=2 \frac{x_{\text {in }}-x_{\min }}{x_{\max }-x_{\min }}-1$,

the contracting limits $\left(x_{\min }-x_{\max }\right)$ being

1. $Z_{\mathrm{H}}:-10-60 \mathrm{dBZ}$,

2. $Z_{\mathrm{DR}}:-1.5-5 \mathrm{~dB}$,

3. $K_{\mathrm{dp}}^{\prime}:-10-7$,

4. $\rho_{\mathrm{hv}}^{\prime}:-50-5.23$.

The fifth parameter is scaled by means of a significantly stricter sigmoid transformation (Eq. 4). In this way, in the pixel assignment, the external parameter literally plays the role of ice/liquid phase indicator (Fig. 2).

The classification itself is performed by determining the Euclidean distance in the five-dimensional space of any observed precipitation pixel with respect to the nine defined centroids (Fig. 6). The distance of the $k$ th observed pixel and the $j$ th centroid is calculated as

$D_{E}^{k, j}=$

$\sqrt{w_{1}\left(\delta Z_{\mathrm{H}}\right)^{2}+w_{2}\left(\delta Z_{\mathrm{DR}}\right)^{2}+w_{3}\left(\delta K_{\mathrm{dp}}^{\prime}\right)^{2}+w_{4}\left(\delta \rho_{\mathrm{hv}}^{\prime}\right)^{2}+w_{5}(\delta \text { Ind })^{2}}$,

with

$\delta x=x_{k}-x_{j}$, 


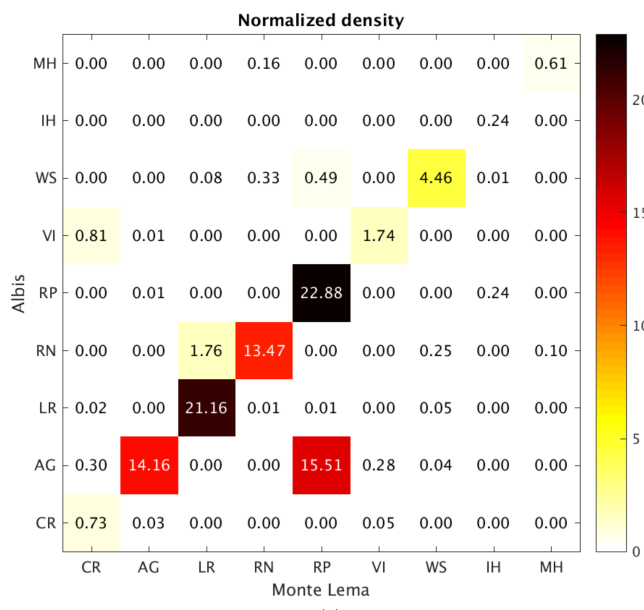

(a)

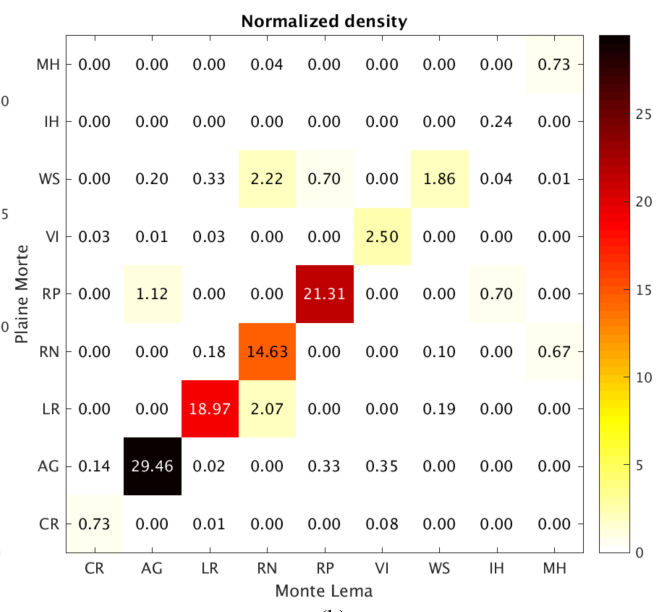

(b)

Figure 7. Matching matrix of the classification results obtained with Monte Lema data with the Monte Lema centroids opposed to the classifications results obtained with the same data but with the centroids of (a) Albis and (b) Plaine Morte. The results are averaged over the entire duration of the convective event that occurred in the proximity of Monte Lema radar site on 12 June 2014.

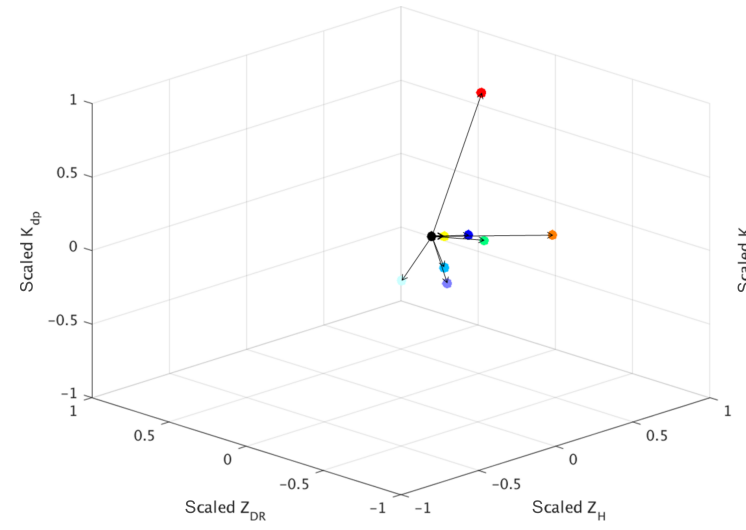

(a)

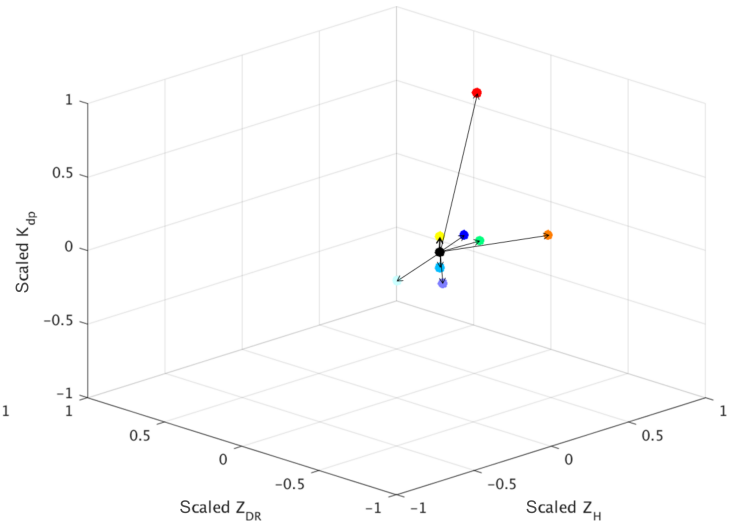

(b)

Figure 8. Example taken from the classification illustrated in Fig. 9: (a) lower entropy is 0.2753 and (b) higher entropy is 0.4756 . Centroids are represented by points of different colour, according to the colour code from Fig. 9, with the observation being represented by a black point. The bold line corresponds to the smallest distance, the arrow indicating the actual label.

where the weights are $w_{i}=1$ for $i=1 \ldots 3$, while $w_{4}=0.75$ and $w_{5} \leq 0.5$. The impact of the $\rho_{\mathrm{hv}}^{\prime}$ parameter is slightly decreased due to the highly probable residual noise in the correlation between channels, whose influence is emphasized with a logarithmic transformation. Out of the nine obtained distances for each pixel, it is the minimal one which determines its hydrometeor label.

The choice of the employed distance was investigated by comparative analysis with the standardised Euclidean distance (including standard deviation) and the Mahalanobis distance (including covariance estimate; Mahalanobis, 1936). By taking into the account the simplicity and the computational efficiency required for the operational purpose, we have adopted the simplest option.

\subsubsection{Entropy}

The obtained classification map is accompanied by a corresponding entropy estimation. The entropy indicates a level of uncertainty with which a hydrometeor class is assigned to an observation. Lower entropy reflects significant confidence in the assigned hydrometeor label, while higher entropy should be interpreted as an uncertain decision and a potential mixtures indicator.

Out of Rényi's entropies (Rényi, 1960), we have chosen the min entropy as a measure of uncertainty. The distances (arrow lines in Fig. 8) of the observation with respect to all centroids are converted to the respective probabilities assuming exponential distribution (emphasising lower distances):

$p_{i}=3 \exp \left(-3 d_{i}\right), \quad i=1, \cdots 8(9)$ 


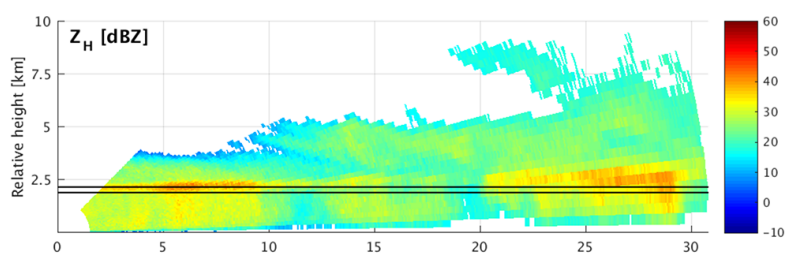

(a)

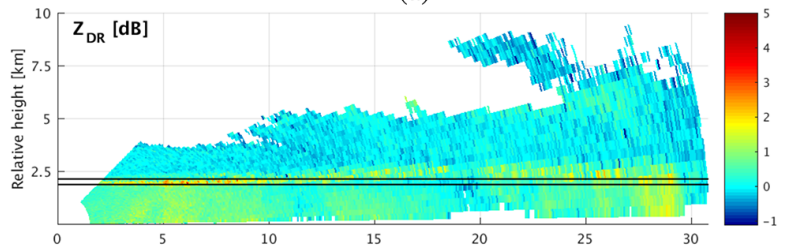

(b)

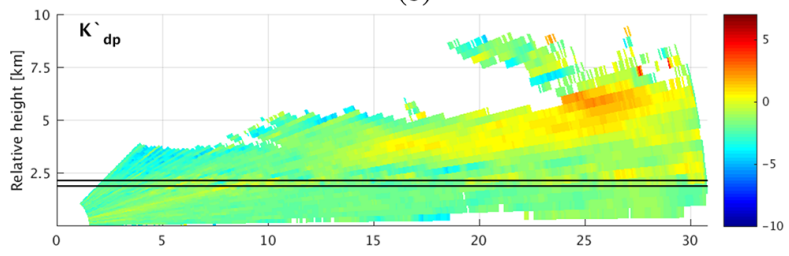

(c)

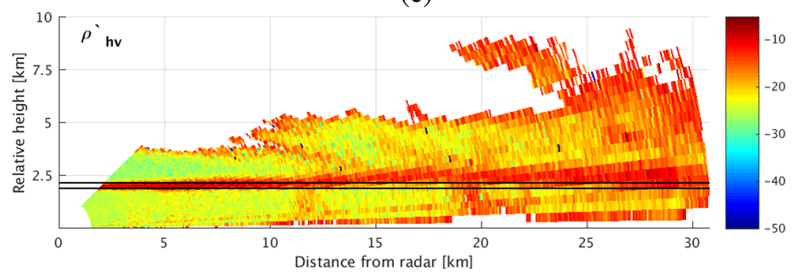

(d)

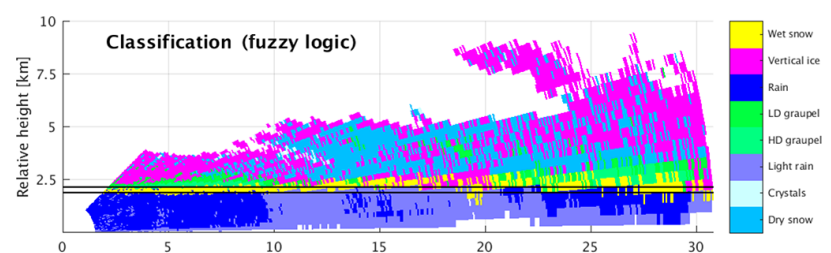

(e)

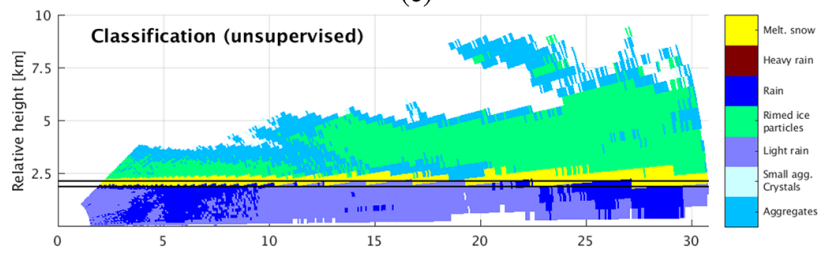

(f)

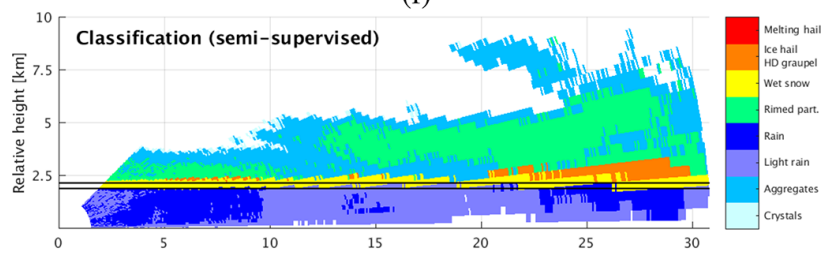

(g)

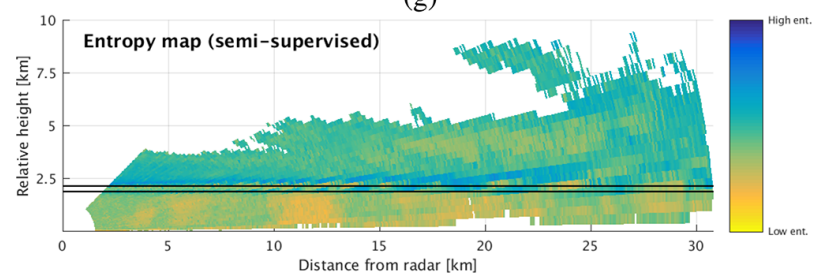

(h)

Figure 9. MXPol RHI profile $226.8^{\circ}$ azimuth, 14:25 UTC, 29 September 2013, in Ardèche, France: (a) $Z_{\mathrm{H}}$, (b) $Z_{\mathrm{DR}}$, (c) $K_{\mathrm{dp}}^{\prime}$, (d) $\rho_{\mathrm{hv}}^{\prime}$. Comparison of semi-supervised approach (g) with its supervised (e) and unsupervised counterpart (f), along with the entropy estimate of the semi-supervised approach (h).

which are further on normalised so that $\sum_{i}^{8(9)} p_{i}=1$. Finally, min entropy is calculated as

$H=-\log _{8(9)} p_{\max }$,

with $p_{\max }$ corresponding to the distance with respect to the nearest centroid (bold arrow line in Fig. 8).

\section{Performance analysis}

The algorithm with the derived sets of centroids for the considered radars has been applied on a number of characteristic events observed by these radars. This is used to illustrate, and to a degree validate, the prospects of the proposed classification.

We start the illustration with a very descriptive comparison, based on the MXPol X-band data. Namely, we compare the proposed method with the classification based on the set of centroids issued from the corresponding unsupervised method (Grazioli et al., 2015), which was in fact defined using the same dataset. Supervised classification based on fuzzy logic was considered as well (modelled upon the work of Dolan and Rutledge, 2009). The example of the comparison shown in Fig. 9 genuinely represents the ensemble of results obtained while treating MXPol data.

The results of semi-supervised classification at $\mathrm{X}$ band somewhat differ from those obtained by the supervised method (54\% of matching after the suitable aggregation of classes) and match to a significant degree those obtained by employing the centroids derived from the unsupervised method ( $83 \%$ of matching). The main difference in the latter comparison can be spotted by closely observing ice phase classes (e.g. crystals, aggregated and rimed ice particles) as well as the wet snow (melting) layer.

However, this does not imply that aside from the automatised centroid derivation a real contribution of the supervised routine to the proposed semi-supervised one does not exist, i.e. constraints introduced in clustering indeed do not improve the decision process. Namely, the centroids derived from the unsupervised method could in a way be taken as reference due to the following reasons: they are derived using a fairly sophisticated clustering method (AHC); the information about the texture is explicitly introduced; the iden- 

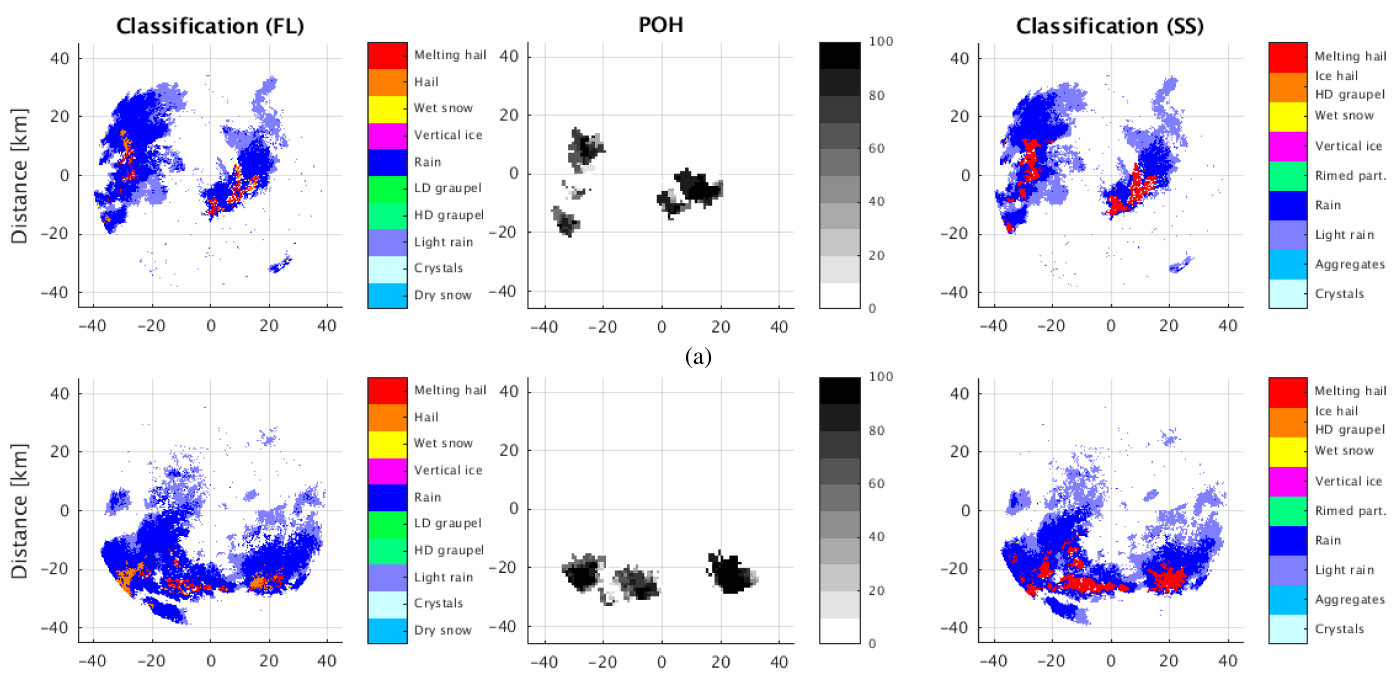

(a)

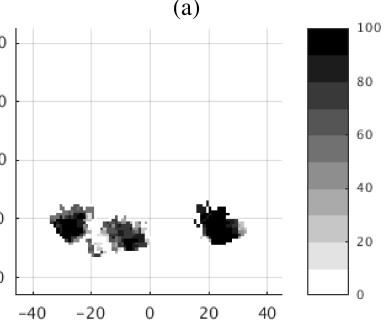

(b)
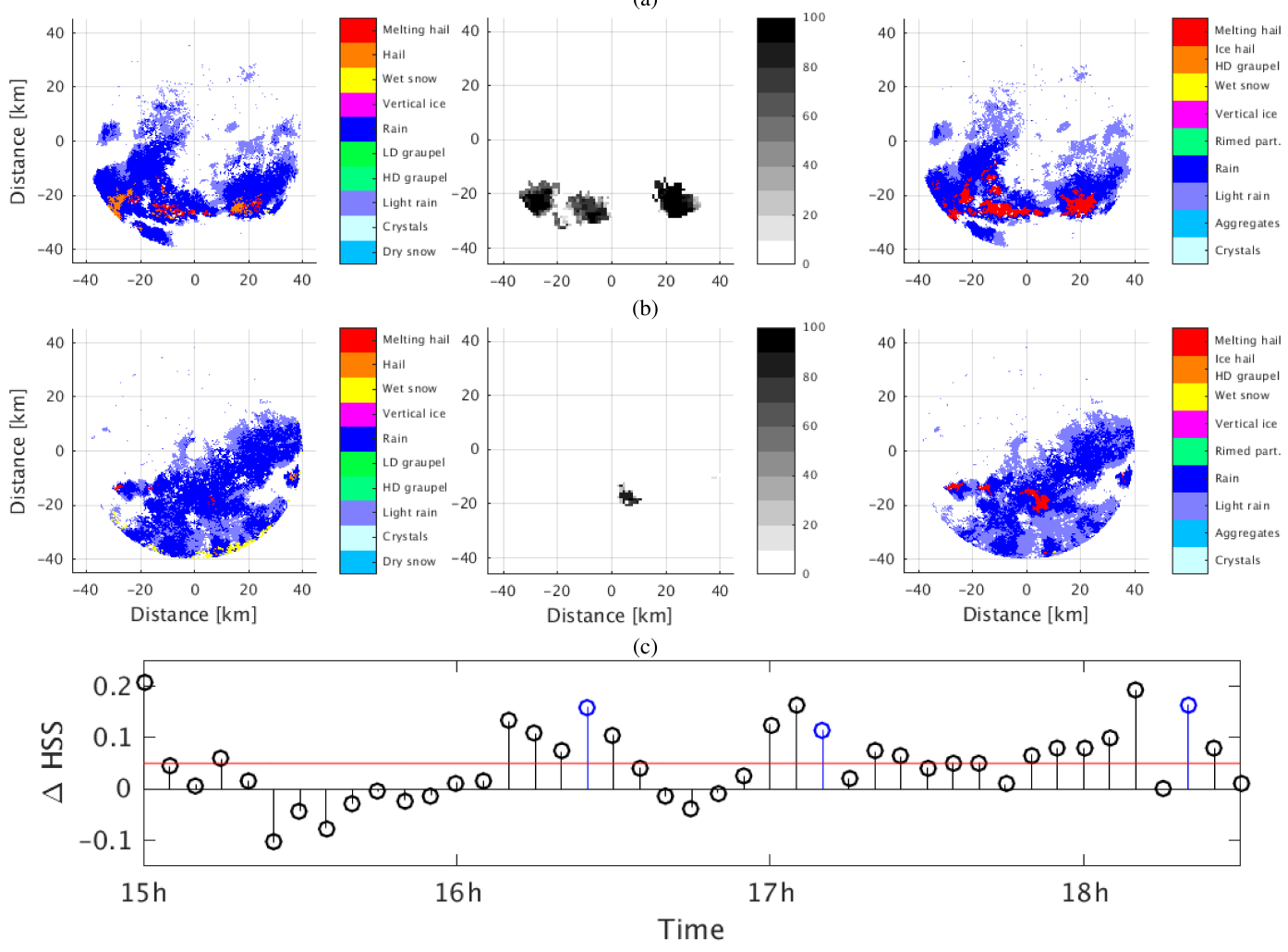

(d)

Figure 10. Comparison of fuzzy-logic-based method (FL) and our semi-supervised method (SS) with the probability of hail (Nisi et al., 2016) - Albis radar, 2.5 $5^{\circ}$ elevation, 12 June 2014: (a) 16:25, (b) 17:10, (c) 18:20; (d) along with the quantitative evaluation of matching ( $\triangle$ HSS $)$, averaged over three elevations $\left(1,1.6\right.$ and $\left.2.5^{\circ}\right)$.

tification is performed through human expertise, using complementary data when possible.

Here, by using the simpler $k$-medoids clustering method and without considering at all the texture information, we obtain very similar results. Furthermore, the identification is performed automatically, modifiable theoretical assumptions are used at the input and more classes are derived.

The entropy estimate illustrated in (Fig. 9d) shows the potential of this parameter to detect radar sampling volumes without dominant hydrometeor type (hydrometeor mixtures), particularly elevated values above the detected melting layer.

These initial indications about the relative performances of the presented method are elaborated by including other $\mathrm{X}$-band and C-band datasets in the frameworks of detection performances, spatial homogeneity and robustness.

\subsection{Detection performance}

The previous comparison with the unsupervised approach, based on the same representative dataset, could be indeed considered as a sort of validation, especially when we take into the account the comprehensive validation of ice particles supported by a two-dimensional video disdrometer (Grazioli et al., 2014). Nevertheless, we have analysed the performances of the proposed semi-supervised method, using the same 2-D video disdrometer dataset (2DVD; Davos, 2010-2011). The analysis was done in the same framework as the one used in Grazioli et al. (2015). The hydrometeor labels assigned to the sampling volumes above the 2DVD are compared to the corresponding labels of hydrometeor populations, as designated by the 2DVD classification 


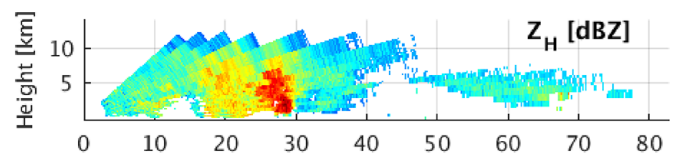

(a)

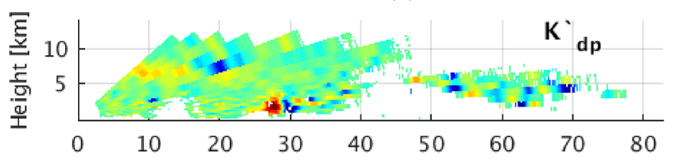

(c)

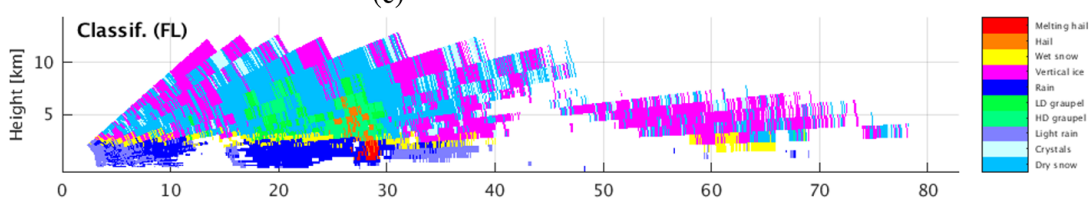

(e)

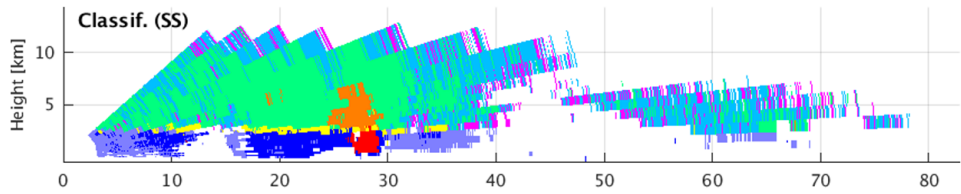

(f)

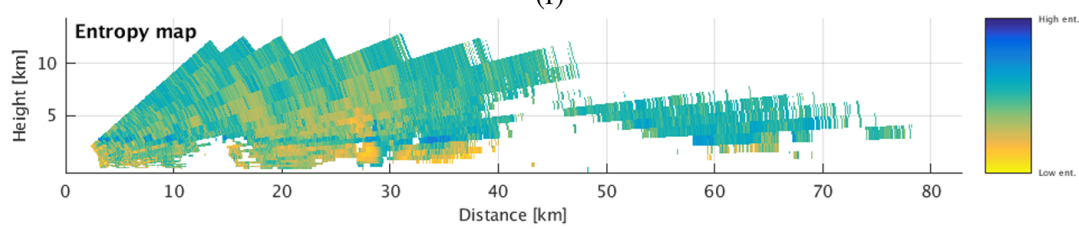

(g)

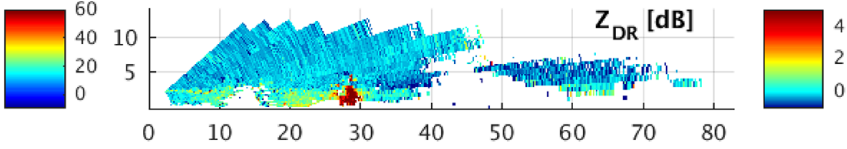

(b)

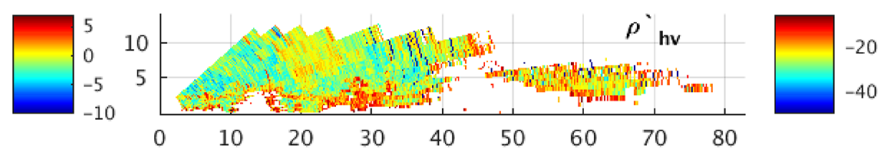

(d)

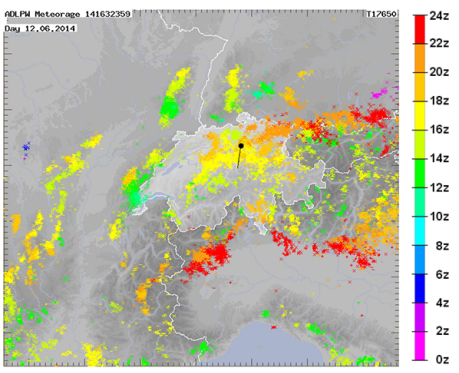

(h)

Figure 11. Hail continuity and vertical ice detection, 17:30, 12 June 2014. Albis reconstructed RHI profile, $187^{\circ}$ azimuth: (a) $Z_{\mathrm{H}}$, (b) $Z_{\mathrm{DR}}$, (c) $K_{\mathrm{dp}}^{\prime}$, (d) $\rho_{\mathrm{hv}}^{\prime}$, (e) FL classification, (f) SS classification, (g) SS measure of entropy. (h) Lightning distribution by time (cloud to ground \pm and intra-clouds indicated with an X) (Météorage.

Table 2. Confusion matrix (semi-supervised method vs. 2DVD).

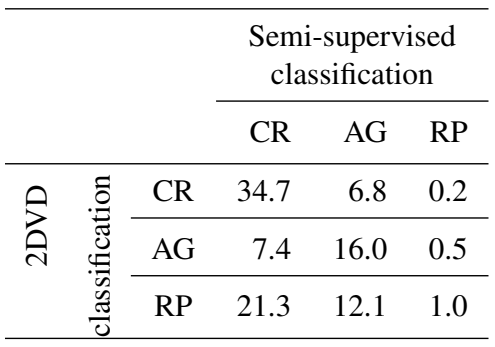

method (Grazioli et al., 2014). After appropriate aggregation of classes (CR is crystals, AG is aggregates and RP is rimed ice particle + ice hail/high density graupel), we obtained the confusion matrix given in Table 2 .

The comparison of Cohen's kappa and overall accuracy with respect to the results given in Grazioli et al. (2015), quantified in Table 3, shows a slight improvement over the unsupervised and an important improvement over the supervised method. The vertical distance of $400 \mathrm{~m}$ between the in-
Table 3. Cohen's kappa and overall accuracy.

\begin{tabular}{lll}
\hline Method & $\begin{array}{l}\text { Cohen's } \\
\text { kappa }\end{array}$ & $\begin{array}{l}\text { overall } \\
\text { accuracy }\end{array}$ \\
\hline Fuzzy logic (Grazioli et al., 2014) & 0.08 & 0.38 \\
Unsupervised (Grazioli et al., 2014) & 0.23 & 0.49 \\
Semi-supervised & 0.25 & 0.52 \\
\hline
\end{tabular}

strument and the lowest radar sampling volume could explain the generally low score values.

The operational radars do not have a sufficient visibility above the location at which the 2DVD was deployed, so a comparison to the ground level reference is not possible. Therefore, we rather concentrate on evaluating the detection of hail and liquid precipitation using $\mathrm{C}$-band data.

The results of the classification at $\mathrm{C}$ band have been compared to the 5 min operational hail detection product - probability of hail (POH) (Nisi et al., 2016). Though also based on radar measurements due to its entirely different concept, this algorithm can be considered as a quasi-independent reference. Namely, the derived POH is proportional to the dif- 

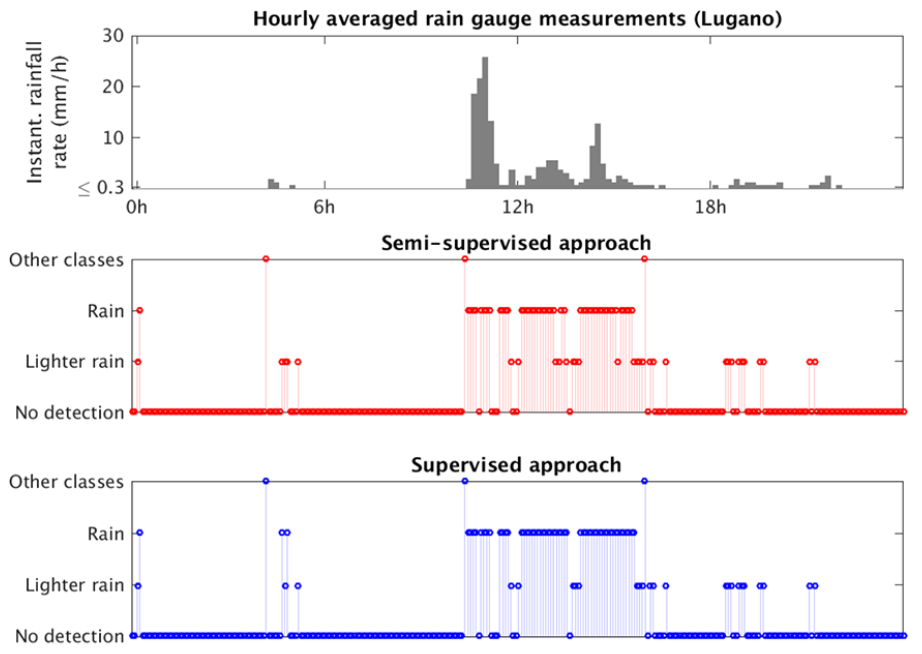

(a)
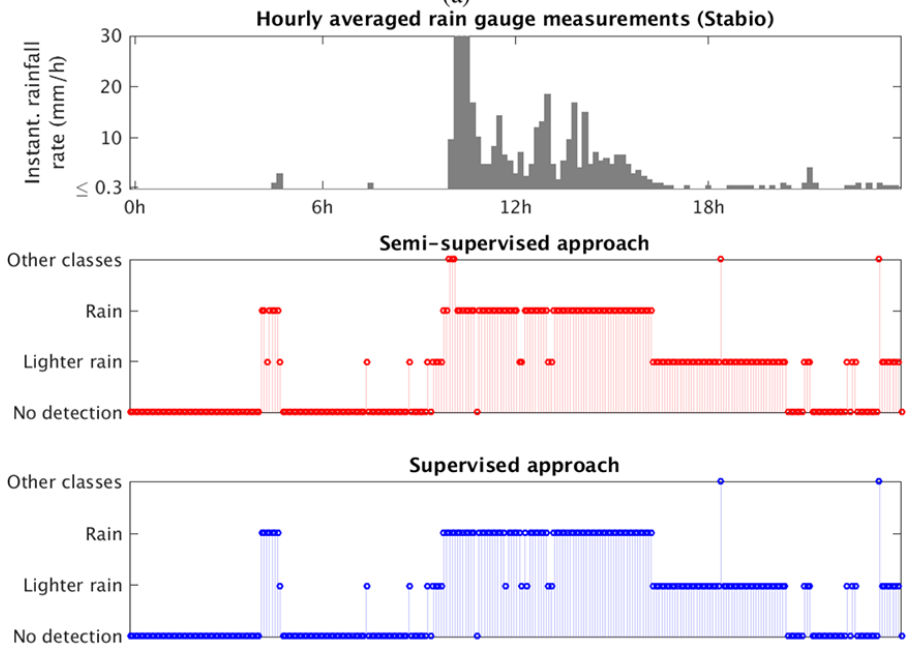

(b)

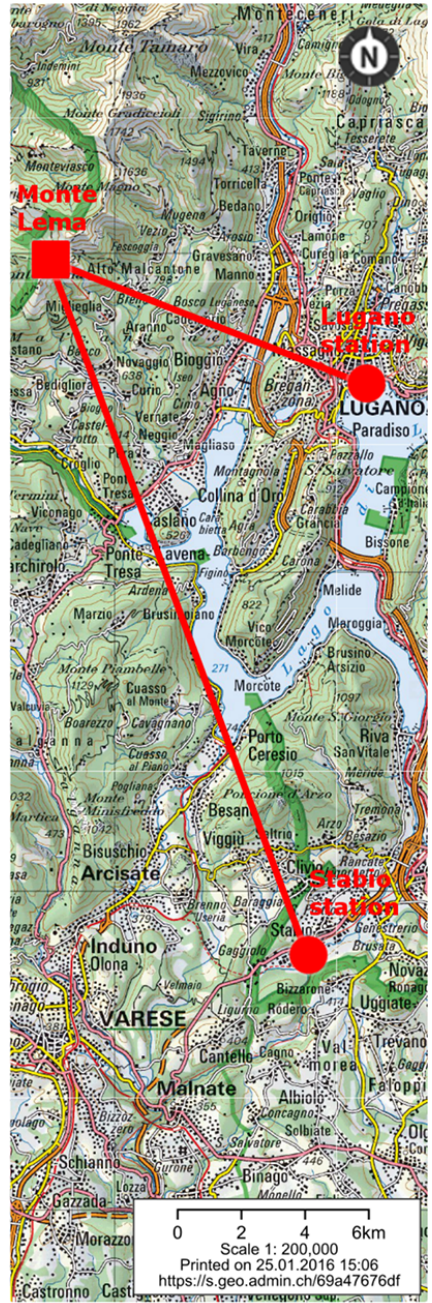

(c)

Figure 12. Comparison of light rain/rain/other classes classification, with the rain-gauge measurements - Monte Lema radar, 14 June 2015 : (a) Lugano station; (b) Stabio station; (c) map of the region ( ${ }^{\circledR}$ Swiss federal authorities, 2007).

ference between $45 \mathrm{dBZ}$ echo top height and $0^{\circ} \mathrm{C}$ isotherm height (Witt et al., 1998; Foote et al., 2005). Differences below $1.65 \mathrm{~km}$ indicate no hail, while those above $5.5 \mathrm{~km}$ mean $100 \%$ hail probability.

The comparison, illustrated in Fig. 10, displays POH product derived using the entire radar volume and the classification results obtained with only one elevation $\left(2.5^{\circ}\right.$, only one horizontal cross section of the convective event). The chosen time steps from the convective event, occurring in the proximity of Albis radar, show a very good matching with the proposed classification method, better than is the case with the supervised fuzzy-logic approach. Quantification of the comparison over a period of time, through Heidke skill score $\left(\Delta \mathrm{HSS}_{\text {elev }}=\mathrm{HSS}_{\text {elev }}(\mathrm{POH}, \mathrm{SS})-\mathrm{HSS}_{\text {elev }}(\mathrm{POH}, \mathrm{FL})\right)$, shows an advantage of the proposed method in terms of matching during the span of the convective event (average value of $\Delta \mathrm{HSS}=0.05$ ). Heidke skill score is calculated between the binarised $\mathrm{POH}$ product $(1$ if $\mathrm{POH}>50 \%, 0$ otherwise) and the binarised classification ( 1 in case of melting or ice hail, 0 otherwise), the latter re-gridded to Cartesian coordinates. $\triangle \mathrm{HSS}$ is averaged over $1,1.6$ and $2.5^{\circ}$ elevation:

$$
\Delta \mathrm{HSS}=\frac{1}{3}\left(\Delta \mathrm{HSS}_{1}{ }^{\circ}+\Delta \mathrm{HSS}_{1.6^{\circ}}+\Delta \mathrm{HSS}_{2.5^{\circ}}\right) .
$$

The division of hail in ice hail and high-density graupel on one hand and melting hail on the other hand (Ryzhkov et al., 2013) allowed us to bypass the obstacle of the fifth parameter (though lower weighted) and properly identify the convective core of the storm, which can be observed in Fig. 11.

The increased presence of the vertically aligned ice could be explained by the reported atmospheric lightning, although a straightforward relation cannot be assumed (Hubbert et al., 2014; Roberto et al., 2016). In terms of vertical ice detection, a comparison with the conventional (fuzzy logic) approach (Fig. 11e) could serve as an indicator of a certain robust- 
ness of the semi-supervised method with respect to the differential attenuation; that is, despite the reported atmospheric lightning, after analysing $Z_{\mathrm{H}}$ it seems more plausible that the observed negative $Z_{\mathrm{DR}}$ (Fig. 11b) is partly a result of the differential attenuation and therefore should not be labelled as vertically aligned ice.

As it was the case with Fig. 9d, Fig. 11g shows an increase in the entropy estimate with the increase in sampling volume size, confirming the potential role of this parameter in hydrometeor mixtures identification. The analysis of the event presented in Fig. 10 (with the maximal range of $120 \mathrm{~km}$ considered) indicates a moderate correlation (Pearson coefficient of correlation: 0.42 ) between entropy and range, the latter being proportional to sampling volume size.

An interesting remark, related to the operational implementability, is that the calculation of the semi-supervised classification of the illustrated reconstructed RHI along with its measure of entropy (Fig. 11e and Fig. 11g) is 6 times faster than the calculation of the corresponding fuzzy-logic counterpart.

An additional comparison with the corresponding supervised routine concerns liquid precipitation (Fig. 12). Namely, $10 \mathrm{~min}$ rain-gauge measurements at two MeteoSwiss stations (in the vicinity of Monte Lema radar) are compared to the rain vs. light rain output of semi-supervised and supervised (fuzzy logic) classification. Although drawing a border line between light rain and rain is indeed somehow debatable, by observing ground measurements one can perceive a very high plausibility of the results obtained with both methods. An advantage of the proposed method, however, remains its computational efficiency: in this particular case, which assumes classifying a $7 \times 7$ volumes around the station of interest, the semi-supervised method output is obtained in average 4.6 times faster.

\subsection{Spatial homogeneity}

The comparison with the output of a fuzzy-logic algorithm, which uses similar membership functions to those employed in constraining our clustering, was quantified using the spatial homogeneity feature, derived from the co-occurrence matrix:

$C(p, q)=$

$\sum_{i} \sum_{j} \begin{cases}1, & \text { if } I(i, j)=p \text { and } I(i \pm 1, j \pm 1)=q, \\ 0, & \text { otherwise }\end{cases}$

where $i, j$ is the position indices and $p, q$ are pixel values (in our case number of a label - from 1 to 9) (Haralick et al., 1973). It is actually a measure of the co-occurrence matrix diagonality:

$\mathrm{SH}=\sum_{p, q} \frac{C(p, q)}{1+|p-q|}$,
Table 4. MXPol X-band radar - comparison of semi-supervised approach with its supervised and unsupervised counterpart, spatial homogeneity.

\begin{tabular}{lll}
\hline Freq. band & Method & $\begin{array}{l}\text { Spatial homogeneity } \\
(\mathrm{SH})\end{array}$ \\
\hline \multirow{3}{*}{$\mathrm{X}$} & Supervised & 0.8037 \\
& Unsupervised & 0.8795 \\
& Semi-supervised & 0.8748 \\
\hline
\end{tabular}

Table 5. Albis C-band radar - comparison of semi-supervised approach with its supervised counterpart, spatial homogeneity.

\begin{tabular}{lll}
\hline Freq. band & Method & $\begin{array}{l}\text { Spatial homogeneity } \\
(\mathrm{SH})\end{array}$ \\
\hline \multirow{2}{*}{$\mathrm{C}$} & Supervised & 0.6506 \\
& Semi-supervised & 0.7179 \\
\hline
\end{tabular}

estimated at $\mathrm{X}$ band for two RHIs $\left(226.8^{\circ}\right.$ and $\left.316.7^{\circ}\right)$, during the event around the instant illustrated in Fig. 9 (14-16 h). As illustrated in Table 4 there is a non-negligible increase in averaged spatial homogeneity with respect to fuzzy logic and very small decrease with respect to the unsupervised approach, which nevertheless contains spatial information and has one class less (advantageous for this sort of comparison).

The spatial homogeneity analysis in an analogous framework was applied in case of C-band data, as well. Namely, the spatial homogeneity scores (Eq. 15) are derived for the reconstructed RHIs around $187^{\circ}$ azimuth angle, presented in Fig. $11\left(186-188^{\circ}\right)$, for each $5 \mathrm{~min}$ instant during the event presented in Fig. 10 (15:00-18:30 UTC). To ensure a fair comparison (equal number of classes), LDG and HDG classes in the fuzzy-logic approach are merged to one. After averaging, the results show an advantage of semi-supervised method over its bin-based counterpart (Table 5).

\subsection{Robustness analysis}

Finally, we performed the matching analysis by applying both our classification, based on MXPol and DX50 centroids, and the fuzzy-logic approach, on two X-band radars pointing toward the same volume (Fig. 13). The analysis is performed using normalised matching matrix (otherwise called confusion matrix), which provides information about the matching of hydrometeor labels in the overlapping sampling volumes of two radars. It is normalised with respect to the total number of observations. The peculiarity of the analysed day is that the MXPol radar had an issue with the magnetron performance, causing a significant reflectivity bias.

Before bias correction, the semi-supervised method shows better matching performances: $55 \%$ of observations on the diagonal vs. $50 \%$ in case of the fuzzy-logic approach. However, more significant is the fact that after bias correction, 


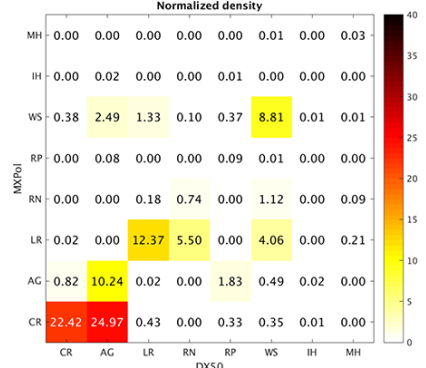

(a1)

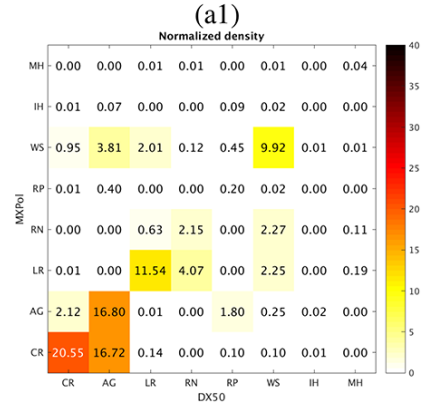

(a2)

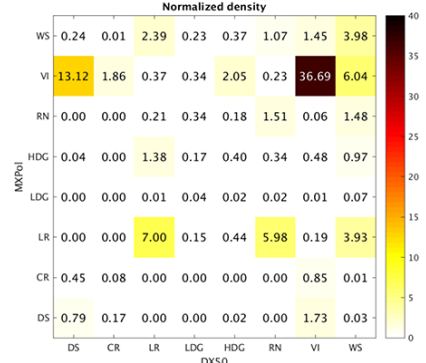

(b1)

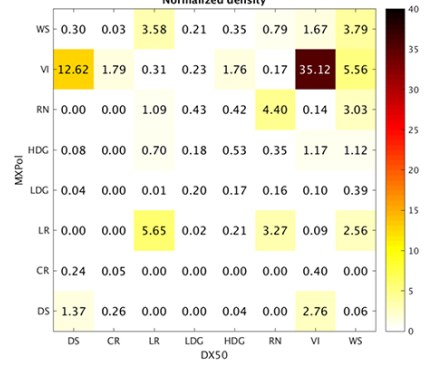

(b2)

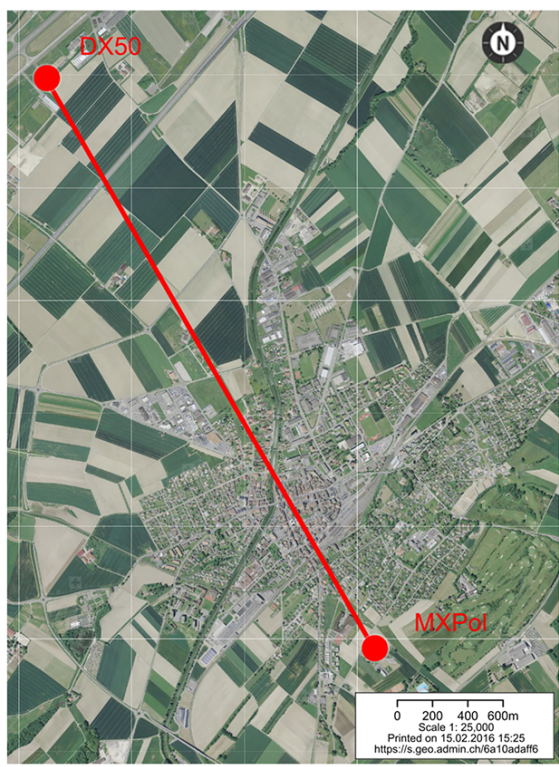

(c)

Figure 13. Intercomparison between classifications of the data acquired by MXPol and DX50 radars, over the town of Payern, $\mathrm{CH}$, on 8 April 2014: (a) normalized matching matrix-semi-supervised classification; (b) normalized mixing matrix-supervised classification; (c) configuration of two radars $\left({ }^{\oplus}\right.$ Swiss federal authorities, 2007). 1 indicates before bias correction; 2 indicates after bias correction.

the performances of the presented method improve significantly more than those of the considered fuzzy-logic approach: $61 \%$ vs. $51 \%$. We deduce that this difference indicates a stronger reliance of the semi-supervised method on the polarimetric radar parameters, whereas the decision of the supervised approach depends significantly on the external (fifth) parameter.

\section{Conclusions and future perspectives}

In this paper, we propose a novel semi-supervised method for hydrometeor classification from polarimetric radar data. The idea is to combine the main advantages of both supervised and unsupervised approaches, while keeping the potential operational implementation reasonably simple. This is achieved through the statistical clustering of representative observations of the considered polarimetric radar. It includes implicitly introduced constraints provided by the state-ofthe-art assumptions, which are appropriately modified using scattering simulations, and enforced by the KS statistical test. The obtained clusters are afterwards merged into nine distinct sets of observations, representing different hydrometeor classes. Each of these classes is characterized by a centroid in the five-dimensional space formed by four polarimetric parameters and liquid/melting/ice phase indicator. The obtained set of centroids is adapted to the considered radar. Their coordinates result from both the distinctive properties of the radar and the microphysical assumptions reflected through the employed parametric distributions of polarimetric vari- ables for different hydrometeor types. The real-time classification itself is performed by examining all the measurements of interest in the evoked five-dimensional space and associating them to the closest centroid in the framework of Euclidean metric. The method is applied on three operational C-band MeteoSwiss radars and two research X-band radars. The comparative analysis with the standard supervised and unsupervised approach was done in order to properly position the proposed method, stating the benefits and the limitations. A meaningfulness of the hydrometeor identification was evaluated using ground truth measurements and wellestablished MeteoSwiss operational products. We illustrated some noteworthy properties of the proposed classification, which concern discrimination between different solid phase particles, hail detection performance and spatial homogeneity of the classification output. Simultaneously, we demonstrated a very low dependency on non-radar inputs and a significantly enhanced computational efficiency.

The presented method is already running in real time on a mobile X-band radar (DX50), while the operational implementation in the processing chain of Rad4Alp network is under progress. This constitutes the foundation for the envisaged long-term statistical evaluation of the method.

For the moment, the reference observations are mostly generated using appropriately adjusted state-of-the-art membership functions. The idea is to replace this input in the future with potentially statistically richer information such as are the EM properties of hydrometeors which have been recently appearing in the literature, determined by employ- 
ing the method of moments (MOM) (Mirkovic et al., 2015) or the invariant imbedding T-matrix method (Pelissier et al., 2015). This way, we would entirely exploit the potential of the KS test, limited in the current implementation by the imposed parametric probability density distributions of the reference observations. Due to the non-availability of recent ground-truth data for the ice phase particles, we had to rely on the framework employed in the validation the unsupervised method from Grazioli et al. (2015). However, with the envisaged campaigns involving a multi-angle snowflake camera (MASC; Garrett et al., 2012), data acquired at the ground level above $0^{\circ}$ isotherm will be used to improve the discrimination between aggregates and rimed particles. Finally, the biggest challenge in front of us would be dealing with the pixels suspected to be hydrometeor mixtures. Therefore, the plan is to deal with the sampling volumes characterized with high entropy (potential hydrometeor mixtures), either through their decomposition or through defining a new set of mixed classes, dominantly for far ranges.

\section{Data availability}

Datasets acquired by MXPol X-band radar can be made publicly available upon request to the authors. Datasets acquired by Rad4Alps C-band operational network can be made publicly available in the re-sampled range resolution $(500 \mathrm{~m}$ rather than $83 \mathrm{~m}$ ) upon request to the authors. Datasets acquired by DX50 X-band radar cannot be made publicly available due to the constraints imposed by a project partner of MeteoSwiss. 


\section{Appendix A: Employed clustering constraints}

The basis of the membership functions employed to generate reference polarimetric observations by means of an inverse transform sampling is been adopted from Dolan et al. (2013) and Dolan and Rutledge (2009) respectively for C and $\mathrm{X}$ bands. They have the form of a bell-shaped function:

$f(x)=\frac{1}{1+\left(\frac{x-m}{a}\right)^{2 b}}$,

with $x$ being a polarimetric parameter and $m, a$ and $b$ being respectively mean, width and slope of a function, provided in Tables A1 and A2. Less rigorous slope criteria were used at X band for the identification rate comparative analysis (Fig. 4).

Wet snow class for X band, not defined in Dolan and Rutledge (2009), was adopted from Grazioli et al. (2015).

Ice and melting hail classes for both considered frequency bands were defined using scattering simulations with a single- and a double-layer T-matrix method (Mishchenko et al., 1996), as indicated in Sect. 3. The same simulator was used for modifications introduced in crystals and vertically aligned ice classes. The modified parameters are emphasized using bold font in Tables A1 and A2. The rationales behind the modifications are the following.
- CR and VI: the very broad $Z_{\mathrm{H}}$ distributions of these two classes (e.g. reflectivity of vertically aligned ice in Cband ranges roughly from -26 to $24 \mathrm{dBZ}$ ) are in discordance with our scattering simulations (indicating significantly more contracted ranges). This could be a consequence of the considered particle size distributions. Basically, in order to avoid an important impact of such broad assumptions on the convergence behaviour of the centroids derivation algorithm, we decided not to account for very small and very big crystals $(D>30 \mathrm{~mm}$ - converging towards aggregates).

- IH and MH: given the specific nature of the IH class, which is directed by the fifth parameter to the high altitudes, this class has more specific properties with respect to the conventional hail class (insignificant liquid water content). Consequently, there is a need for the complementary MH class.

Table A1. C-band membership function parameterisation. The modified parameters are emphasized using bold font.

\begin{tabular}{lrrr|rr|rr|rr|rrr}
\hline \multirow{2}{*}{ HT } & \multicolumn{3}{c|}{$Z_{\mathrm{H}}(\mathrm{dBZ})$} & \multicolumn{3}{|c|}{$Z_{\mathrm{DR}}(\mathrm{dB})$} & \multicolumn{3}{|c|}{$K_{\mathrm{dp}}\left(\mathrm{deg} \mathrm{km}^{-1}\right)$} & \multicolumn{3}{c}{$\rho_{\mathrm{hv}}$} \\
& $m$ & $a$ & $b$ & $m$ & $a$ & $b$ & $m$ & $a$ & $b$ & $m$ & $a$ & $b$ \\
\hline CR & -2.8 & $\mathbf{1 2}$ & $\mathbf{5}$ & 2.9 & 2.7 & 10 & 0.08 & 0.08 & 6 & 0.98 & 0.025 & 3 \\
AG & 17 & 18.1 & 10 & 1 & 1.1 & 7 & -0.008 & 0.3 & 1 & 0.93 & 0.07 & 3 \\
LR & 1.75 & 29 & 10 & 0.46 & 0.46 & 5 & 0.03 & 0.03 & 2 & 1 & 0.018 & 3 \\
RN & 39 & 19 & 10 & 2.3 & 2.2 & 9 & 5.5 & 5.5 & 10 & 1 & 0.025 & 3 \\
RP & 37 & 9.2 & 0.8 & 0.9 & 0.9 & 6 & 0.1 & 0.08 & 3 & 1 & 0.025 & 1 \\
VI & -1 & $\mathbf{1 1}$ & $\mathbf{5}$ & -0.9 & 0.9 & 10 & -0.75 & 0.75 & 30 & 0.975 & 0.022 & 3 \\
WS & 24 & 21.3 & 10 & 1.3 & 0.9 & 10 & 0.25 & 0.43 & 6 & 0.8 & 0.10 & 10 \\
MH & $\mathbf{5 8 . 1 8}$ & $\mathbf{8}$ & $\mathbf{1 0}$ & $\mathbf{2 . 1 9}$ & $\mathbf{1 . 5}$ & $\mathbf{1 0}$ & $\mathbf{1 . 0 8}$ & $\mathbf{2}$ & $\mathbf{6}$ & $\mathbf{0 . 9 5}$ & $\mathbf{0 . 0 5}$ & $\mathbf{3}$ \\
IH & $\mathbf{4 8 . 8}$ & $\mathbf{8}$ & $\mathbf{1 0}$ & $\mathbf{0 . 3 6}$ & $\mathbf{0 . 5}$ & $\mathbf{1 0}$ & $\mathbf{0 . 0 7}$ & $\mathbf{0 . 1 5}$ & $\mathbf{6}$ & $\mathbf{0 . 9 9}$ & $\mathbf{0 . 0 5}$ & $\mathbf{3}$ \\
\hline
\end{tabular}

Table A2. X-band membership function parameterisation. The modified parameters are emphasized using bold font.

\begin{tabular}{lrrr|rrr|rrr|rrrr}
\hline \multirow{2}{*}{ HT } & \multicolumn{3}{c|}{$Z_{\mathrm{H}}(\mathrm{dBZ})$} & \multicolumn{3}{|c|}{$Z_{\mathrm{DR}}(\mathrm{dB})$} & \multicolumn{3}{|c|}{$K_{\mathrm{dp}}\left(\mathrm{deg} \mathrm{km}^{-1}\right)$} & \multicolumn{3}{|c}{$\rho_{\mathrm{hv}}$} \\
& $m$ & $a$ & $b$ & $m$ & $a$ & $b$ & $m$ & $a$ & $b$ & $m$ & $a$ & $b$ \\
\hline CR & -3 & $\mathbf{1 2}$ & $\mathbf{5}$ & 3.2 & 2.6 & 10 & 0.15 & 0.15 & 6 & 0.985 & 0.015 & 3 \\
AG & 16 & 17 & 10 & 0.7 & 0.7 & 7 & 0.2 & 0.2 & 1 & 0.989 & 0.011 & 3 \\
LR & 2 & 29 & 10 & 0.5 & 0.5 & 5 & 0.18 & 0.18 & 2 & 0.992 & 0.007 & 3 \\
RN & 42 & 17 & 10 & 2.7 & 2.8 & 9 & 12.6 & 12.9 & 10 & 0.99 & 0.01 & 3 \\
RP & 34 & 10 & 0.8 & 0.3 & 1 & 6 & 0.7 & 2.1 & 3 & 0.993 & 0.007 & 1 \\
VI & 3.5 & $\mathbf{1 4}$ & $\mathbf{5}$ & -0.8 & 1.3 & 10 & -0.1 & 0.08 & 30 & 0.965 & 0.035 & 3 \\
WS & 30 & 20 & 10 & 2.2 & 1.4 & 10 & 1 & 1 & 6 & 0.835 & 0.135 & 10 \\
MH & $\mathbf{5 3 . 3 7}$ & $\mathbf{8}$ & $\mathbf{1 0}$ & $\mathbf{2 . 6}$ & $\mathbf{1 . 5}$ & $\mathbf{1 0}$ & $\mathbf{1 . 3 7}$ & $\mathbf{2}$ & $\mathbf{6}$ & $\mathbf{0 . 9 6}$ & $\mathbf{0 . 0 5}$ & $\mathbf{3}$ \\
IH & $\mathbf{4 5 . 5}$ & $\mathbf{8}$ & $\mathbf{1 0}$ & $\mathbf{- 0 . 0 3}$ & $\mathbf{0 . 5}$ & $\mathbf{1 0}$ & $\mathbf{0 . 1}$ & $\mathbf{0 . 1 5}$ & $\mathbf{6}$ & $\mathbf{0 . 9 7}$ & $\mathbf{0 . 0 5}$ & $\mathbf{3}$ \\
\hline
\end{tabular}


In what concerns the choice of the particle size distribution (PSD) and the aspect ratios for the simulated particles, we opted for the following.

- CR and VI: PSD as the simplified (exponential) version of the empirical, temperature $(T)$-dependent distributions found in Heymsfield et al. (2013):

$$
N(D)=N_{0} \exp -\lambda D
$$

with $\quad N_{0}=3.304 \exp (-0.04607 T), \quad$ and $\quad \lambda=$ $15.3 \exp (-0.053 T)$ for stratiform and $\lambda=$ $3.4 \exp (-0.083 T)$ for convective events.

Aspect ratio follows the empirical relation $h=a D_{\max }^{b}$, with $h$ being the smallest dimension and $D_{\max }$ the largest: spherical ice crystals $(a=0.9, b=1)$, solid thick plates $(a=0.23, b=0.778)$ and dendrites $(a=$ $0.0418, b=0.377$ ) (Matrosov et al., 1996; Pruppacher and Klett, 1997).

- IH and MH: PSD as defined in Cheng et al. (1985):

$$
N(D)=C \Lambda^{4.11} \exp (-\Lambda D),
$$

with $\Lambda$ varying from 0.1 to $1 \mathrm{~mm}^{-1}$ and $\mathrm{C}$ varying from 60 to 300 .

Aspect ratio as defined by Ryzhkov et al. (2011) (dh is dry/ice hail; $\mathrm{mh}$ is melting hail) is

$r_{\mathrm{dh}}= \begin{cases}1-0.02 D, & D \leq 10 \mathrm{~mm} \\ 0.8, & D \geq 10 \mathrm{~mm}\end{cases}$

$r_{\mathrm{mh}}=$

$\begin{cases}r_{\mathrm{dh}}-5\left(r_{\mathrm{dh}}-0.8\right) f_{\mathrm{mw}}, & f_{\mathrm{mw}}<0.2, \\ 0.88-0.40 f_{\mathrm{mw}}, & D 0.2 \leq f_{\mathrm{mw}} \leq 0.8, \\ 2.8-4 r_{w}+5\left(r_{w}-0.56\right) f_{\mathrm{mw}}, & D f_{\mathrm{mw}}>0.8,\end{cases}$

with $f_{\mathrm{mw}}$ being the mass water fraction and $r_{w}$ the aspect ratio of the equivalent raindrop.

With the exception of the modifications explained above, the microphysical properties of the classes correspond to the definitions found in Dolan et al. (2013), Dolan and Rutledge (2009) and Grazioli et al. (2015).

The fifth parameter - a liquid/melting/ice indicator - was generated using the following trapezoidal function:

$f(v)= \begin{cases}0, & \text { if } v<v_{1}, \\ \frac{v-v_{1}}{v_{2}-v_{1}}, & \text { if } v_{1}<v \leq v_{2}, \\ 1, & \text { if } v_{2}<v \leq v_{3}, \\ \frac{v_{4}-v}{v_{4}-v_{3}}, & \text { if } v_{3}<v \leq v_{4}, \\ 0, & \text { if } v>v_{4},\end{cases}$

where the parameters $v_{1}, v_{2}, v_{3}$ and $v_{4}$ are provided in Table A3. An important remark would be that in the implementation of a fuzzy-logic approach, for the purpose of a coherent comparison, instead of the original temperature membership functions, we employed the relative altitude as provided by Grazioli et al. (2015).
Table A3. Trapezoid membership function parameterisation for both $\mathrm{C}$ and $\mathrm{X}$ bands.

\begin{tabular}{lrrrr}
\hline \multirow{4}{*}{ HT } & \multicolumn{4}{c}{$\Delta H(m)$} \\
& $v_{1}$ & $v_{2}$ & $v_{3}$ & $v_{4}$ \\
\hline CR & 0 & 1000 & 2200 & 2500 \\
AG & 0 & 500 & 2000 & 2500 \\
LR & -2500 & -2200 & -300 & 0 \\
RN & -2500 & -2200 & -300 & 0 \\
RP & 0 & 500 & 2000 & 2200 \\
VI & 0 & 1000 & 2200 & 2500 \\
WS & -500 & -300 & 300 & 500 \\
MH & -2500 & -2200 & -300 & 0 \\
IH & 0 & 500 & 2000 & 2500 \\
\hline
\end{tabular}

The employed inference is

$$
\begin{gathered}
\mu_{i}=\frac{w_{Z_{\mathrm{DR}, i}} \beta_{Z_{\mathrm{DR}, i}}+w_{K_{\mathrm{dp}, i}} \beta_{K_{\mathrm{dp}, i}}+w_{\rho_{\mathrm{hv}}, i} \beta_{\rho_{\mathrm{hv}}, i}}{w_{Z_{\mathrm{DR}, i}}+w_{K_{\mathrm{dp}}, i}+w_{\rho_{\mathrm{hv}}, i}} \\
\beta_{Z_{H}, i} T_{\Delta H, i},
\end{gathered}
$$

where three weighting coefficients have the values $w_{Z_{\mathrm{DR}}, i}=$ $w_{K_{\mathrm{dp}}, i}=1$ and $w_{\rho_{\mathrm{hv}}, i}=0.75$. This does not correspond to the weighting coefficients found in the original publications and was chosen for the purpose of the coherent comparison with the presented method. Namely, these are the weights used in the semi-supervised approach, except for the nonradar (fifth) parameter, whose influence is not minimised in the fuzzy-logic approach. 


\section{Appendix B: Data processing}

Table B1. Standard deviation of the error, with the Sun radiation as a reference, without noise subtraction.

\begin{tabular}{l|r|r|r}
\hline Radar & $H / V(\mathrm{~dB})$ & $H /$ Sun $(\mathrm{dB})$ & $V /$ Sun $(\mathrm{dB})$ \\
\hline Albis & \pm 0.04 & \pm 0.12 & \pm 0.10 \\
Monte Lema & \pm 0.07 & \pm 0.12 & \pm 0.13 \\
Plaine Morte & \pm 0.21 & \pm 0.18 & \pm 0.07 \\
\hline
\end{tabular}

Attenuation and differential attenuation for all datasets were corrected in the entire volume using $\mathrm{ZPHI}$ method from Testud et al. (2000), while noise in correlation was corrected according to the standard operational procedure:

$\rho_{\mathrm{hv}}^{\text {corr }}=\rho_{\mathrm{hv}} \sqrt{\left(1+\frac{1}{S / N^{\operatorname{lin}}}\right)\left(1+\frac{Z_{\mathrm{DR}}^{\operatorname{lin}}}{\alpha S / N^{\operatorname{lin}}}\right)}$,

with $\alpha$ being the noise ratio between two channels.

At $\mathrm{C}$ band, instead of using the specific differential phase shift derived as a product of operational Rad4Alp radar network, this parameter was estimated in the presented study by rigorously employing a multi-step approach (Vulpiani et al., 2012) in order to minimise the number of outliers, reinforced by median filtering. In case of the MXPol radar, the method proposed in Schneebeli et al. (2014) is employed, while the specific differential phase for the DX50 radar has been derived using a routine provided by the manufacturer, based on the FIR filtering of differential propagation phase (Hubbert and Bringi, 1995) and a linear regression in deriving $K_{\mathrm{dp}}$.
Concerning the $\mathrm{C}$-band data, due to the tremendous efforts invested in automatic calibration and monitoring of the network, we are confident that the probability of radar errors is significantly reduced (Germann et al., 2015). This is demonstrated in the analysis of $H / V, H /$ Sun and $V /$ Sun errors elaborated in Gabella et al. (2016) and briefly summarised in Table B1.

The same applies to the X-band radars, where careful calibration and monitoring are very important due to their very specific research and operational tasks. 
Acknowledgements. With great appreciation of their very useful comments and suggestions, the authors would like to thank to four anonymous reviewers. The authors would also like to thank their colleagues at LTE and the Radar, Satellite, Nowcasting teams for numerous very useful advices in shaping the presented method. Particularly, we would like to emphasise the help of Marco Boscacci and Daniel Wolfensberger, whose support was very handy in dealing with such a diverse dataset as the one used in the presented research. Last but not the least, we would like to acknowledge the beneficial advice of Andreas Langousis concerning the employed statistical apparatus.

Edited by: G. Vulpiani

Reviewed by: four anonymous referees

\section{References}

Al-Sakka, H., Boumahmoud, A.-A., Fradon, B., Frasier, S. J., and Tabary, P.: A New Fuzzy Logic Hydrometeor Classification Scheme Applied to the French X-, C-, and S-Band Polarimetric Radars, J. Atmos. Ocean. Technol., 52, 2328-2344, 2013.

Baldauf, M., Seifert, A., Forstner, J., Majewski, D., Raschendorfer, M., and Reinhardt, T.: Operational Convective-Scale Numerical Weather Prediction with the COSMO Model: Description and Sensitivities, Mon. Wea. Rev., 139, 3887-3905, 2011.

Bechini, R. and Chandrasekar, V.: A Semisupervised Robust Hydrometeor Classification Method for Dual-Polarization Radar Applications, J. Atmos. Ocean. Technol., 32, 22-47, 2015.

Bringi, V. N., Thurai, R., and Hannesen, R.: Dual-Polarization Weather Radar Handbook, AMS-Gematronik GmbH, 2007.

Chandrasekar, V., Keranen, R., Lim, S., and D., M.: Recent advances in classification of observations from dual polarization weather radars, Atmos. Res., 119, 9-111, 2013.

Cheng, L., English, M., and Wong, R.: Hailstone size distributions and their relationship to storm thermodynamics, J. Climate Appl. Meteor., 24, 1059-1067, 1985.

Dolan, B. and Rutledge, S. A.: A Theory-Based Hydrometeor Identification Algorithm for X-Band Polarimetric Radars, J. Atmos. Ocean. Technol., 26, 2071-2088, 2009.

Dolan, B., Rutledge, S. A., Lim, S., Chandrasekar, V., and Thurai, M.: A Robust C-Band Hydrometeor Identification Algorithm and Application to a Long-Term Polarimetric Radar Dataset, J. Atmos. Ocean. Technol., 52, 2162-2186, 2013.

El-Magd, A., Chandrasekar, V., Bringi, V., and Strapp, W.: Multiparameter radar and in situ aircraft observation of graupel and hail, IEEE T. Geosci. Remote Sens., 38, 570-578, 2000.

Foote, G. B., Krauss, T. W., and Makitov, V.: Hail metrics using convectional radar, in: In Proceedings of 16th Conference on Planned and Inadvertent Weather Modification, 10-13 January 2005, San Diego, CA, USA, Amer. Meteor. Soc., Boston, MA, 2005.

Gabella, M., Boscacci, M., Sartori, M., and Germann, U.: Calibration accuracy of the dual-polarization receivers of the C-band Swiss weather radar network, Atmosphere, 7, 76, 2016.

Garrett, T. J., Fallgatter, C., Shkurko, K., and Howlett, D.: Fall speed measurement and high-resolution multi-angle photography of hydrometeors in free fall, Atmos. Meas. Tech., 5, 26252633, doi:10.5194/amt-5-2625-2012, 2012.
Germann, U. and Joss, J.: Weather radar: Principles and advanced applications, vol. XVII of Physics of Earth and space environment, chap. Operational measurement of precipitation in mountainous terrain, 52-77, Springer Verlag, Heidelberg, Germany, ISBN: 978-3-540-00328-1, 2004.

Germann, U., Boscacci, M., Gabella, M., and Sartori, M.: Peak performance: Radar design for prediction in the Swiss Alps, Meteorological Technology International, 42-45, 2015.

Gibbons, J. D. and Chakraborti, S.: Nonparametric Statistical Inference, Chapman \& Hall/CRC Press, Taylor \& Francis Group, Boca Raton, FL, USA, 5 edn., ISBN 9781420077612, 2011.

Grazioli, J., Tuia, D., Monhart, S., Schneebeli, M., Raupach, T., and Berne, A.: Hydrometeor classification from two-dimensional video disdrometer data, Atmos. Meas. Tech., 7, 2869-2882, doi:10.5194/amt-7-2869-2014, 2014.

Grazioli, J., Tuia, D., and Berne, A.: Hydrometeor classification from polarimetric radar measurements: a clustering approach, Atmos. Meas. Tech., 8, 149-170, doi:10.5194/amt-8-149-2015, 2015.

Hall, M., Goddard, J., and Cherry, S.: Identification of hydrometeors and other targets by dual-polarization radar, Radio Sci., 19, 132140, 1984.

Haralick, R. M., Shanmugam, K., and Dinstein, I.: Textural Features for Image Classification, IEEE T. Syst. Man Cyb., 6, 610-621, 1973.

Heymsfield, A. J., Schmitt, C., and Bansemer, A.: Ice Cloud Particle Size Distributions and Pressure-Dependent Terminal Velocities from In Situ Observations at Temperatures from 0 to $-86^{\circ} \mathrm{C}$, J. Atmos. Sci., 70, 4123-4154, 2013.

Hubbert, J. and Bringi, V. N.: An Iterative Filtering Technique for the Analysis of Copolar Differential Phase and Dual-Frequency Radar Measurements, J. Atmos. Ocean Tech., 12, 643-648, 1995.

Hubbert, J. C., Ellis, S. M., Chang, W.-Y., Rutledge, S., and Dixon, M.: Modeling and interpretation of S-band ice crystal depolarization signatures from data obtained by simultaneously transmitting horizontally and vertically polarized, J. Appl. Meteor. Climatol., 53, 1659-1677, 2014.

Ihler, A. and Mandel, M.: Kernel Density Estimation Toolbox for MATLAB (R13), available at: http://www.ics.uci.edu/ ihler/ code/kde.html (last access: 5 September 2016), 2003.

Kaufman, L. and Rousseeuw, P.: Statistical Data Analysis Based on the $\ell_{1}$-norm and Related Methods, chap. Clustering by means of Medoids, pp. 405-416, North-Holland, 1987.

Kaufman, L. and Rousseeuw, P. J.: Finding Groups in Data: An Introduction to Cluster Analysis, John Wiley \& Sons, Inc., Hoboken, NJ, USA, 368 pp., ISBN 978-0-470-31748-8, 2009.

Kolmogorov, A.: Sulla determinazione empirica di una legge di distribuzione, G. Ist. Ital. Attuari, 4, 83-91, 1933.

Lim, S., Chandrasekar, V., and Bringi, V.: Hydrometeor classification system using dual-polarization radar measurements: model improvements and in situ verification, IEEE T. Geosci. Remote Sens., 43, 792-801, 2005.

Liu, H. and Chandrasekar, V.: Classification of hydrometeors based on polarimetric radar measurements: development of fuzzy logic and neurofuzzy systems, and in situ verification, J. Atmos. Ocean. Technol., 17, 140-164, 2000.

Lloyd., S. P.: Least squares quantization in PCM, IEEE T. Geosci. Remote Sens., 28, 129-137, 1982. 
Mahalanobis, P. C.: On the generalised distance in statistics, Proceedings of the National Institute of Sciences of India, 2, 49-55, 1936.

Marzano, F., Scaranari, D., and Vulpiani, G.: Supervised FuzzyLogic Classification of Hydrometeors Using C-Band Weather Radars, IEEE T. Geosci. Remote Sens., 45, 3784-3799, 2007.

Marzano, F., Botta, G., and Montopoli, M.: Iterative Bayesian Retrieval of Hydrometeor Content From X-Band Polarimetric Weather Radar, IEEE T. Geosci. Remote Sens., 48, 3059-3074, 2010.

MathWorks: Statistics and machine learning toolbox ${ }^{\mathrm{TM}}$ : User's guide (R2015B), The MathWorks, Inc., Natick, MA, US, 2015.

Matrosov, S. Y., Reinking, R. F., Kropfli, R. A., and Bartram, B. W.: Estimation of Ice Hydrometeor Types and Shapes from Radar Polarization Measurements, J. Atmos. Ocean. Technol., 13, 8596, 1996.

Mirkovic, D., Zrnic, D., and Ryzhkov, A.: Full wave electromagnetic approach to the calculation of polarimetric variables for ensembles of rough and nonspheroidal hydrometeors, in: 37th AMS Conf. on Radar Meteorology, 14-18 September 2015, Amer. Meteor. Soc., Norman, OK, USA, 2015.

Mishchenko, M., Travis, L., and Mackowski, D.: T-matrix computations of light scattering by nonspherical particles: a review, J. Quant. Spectrosc. Ra., 55, 535-575, 1996.

Nisi, L., Martius, O., Hering, A., Kunz, M., and Germann, U.: Spatial and temporal distribution of hailstorms in the Alpine region: a long-term, high resolution, radar-based analysis, Q. J. Roy. Meteor. Soc., 142, 1590-1604, doi:10.1002/qj.2771, 2016.

Park, H.-S. and Jun, C.-H.: A simple and fast algorithm for Kmedoids clustering, Expert Syst. Appl., 36, 3336-3341, 2009.

Parzen, E.: On estimation of a probability density function and mode, Ann. Math. Stat., 33, 1065-1076, 1962.

Pearson, E. and Hartley, H.: Biometrika tables for statisticians vol. 2, Cambridge University Press, Cambridge, UK, ISBN 9780521069373, 1972.

Pelissier, C., Kwo-Sen, K., and Clune, T.: Characteristics of the Invariant Imbedding T-Matrix Method for Hydrometeor Scattering Calculations, in: 37th AMS Conf. on Radar Meteorology, 14-18 September 2015, Amer. Meteor. Soc., Norman, OK, 2015.

Pruppacher, H. R. and Klett, J. D.: Microphysics of Clouds and Precipitation, Kluwer Academic Publishers, 2nd edn., ISBN 978$0792344094,1997$.

Rényi, A.: On measures of information and entropy, in: Proceedings of the fourth Berkeley Symposium on Mathematics, Statistics and Probability, University of California Press, Berkeley, CA, USA, 547-561, 1960.

Roberto, N., Adirosi, E., Baldini, L., Casella, D., Dietrich, S., Gatlin, P., Panegrossi, G., Petracca, M., Sanò, P., and Tokay, A.: Multi-sensor analysis of convective activity in central Italy during the HyMeX SOP 1.1, Atmos. Meas. Tech., 9, 535-552, doi:10.5194/amt-9-535-2016, 2016.

Ryzhkov, A., Schuur, T., Burgess, B., Heinselman, P., Giangrande, S., and Zrnic, D.: The joint polarization experiment polarimetric rainfall measurements and hydrometeor classification, B. Am. Meteorol. Soc., 86, 809-824, 2005.
Ryzhkov, A., Pinsky, M., Pokrovsky, A., and Khain, A.: Polarimetric Radar Observations Operator for a Cloud Model with Spectral Microphysics, J. Appl. Meteor. Climatol., 50, 873-894, 2011.

Ryzhkov, A. V., Kumjian, M. R., Ganson, S. M., and Khain, A. P.: Polarimetric Radar Characteristics of Melting Hail. Part I: Theoretical Simulations Using Spectral Microphysical Modeling, J. Appl. Meteor. Climatol., 52, 2849-2870, 2013.

Schneebeli, M., Grazioli, J., and Berne, A.: Improved Estimation of the Specific Differential Phase Shift Using a Compilation of Kalman Filter Ensembles, IEEE T. Geosci. Remote Sens., 52, 5137-5149, 2014.

Seliga, T. A. and Bringi, V. N.: Potential Use of Radar Differential Reflectivity Measurements at Orthogonal Polarizations for Measuring Precipitation, J. Appl. Meteorol., 15, 69-76, 1976.

Smirnov, N.: Table for estimating the goodness of fit of empirical distributions, Ann. Math. Stat., 19, 279-281, 1948.

Snedecor, G. W. and Cochran, W. G.: Statistical Methods, Iowa State University Press, ISBN 978-0813815619, 1989.

Straka, J. and Zrnic, D.: An algorithm to deduce hydrometeor types and contents from multi-parameter radar data, in: Preprints, 26th AMS Conf. on Radar Meteorology, 24-18 May 1993, Norman, OK, USA, Amer. Meteor. Soc., 513-516, Boston, MA, 1993.

Straka, J. M., Zrnic, D. S., and Ryzhkov, A. V.: Bulk Hydrometeor Classification and Quantification Using Polarimetric Radar Data: Synthesis of Relations, J. Appl. Meteor., 39, 1341-1372, 2000.

Testud, J., Le Bouar, E., Obligis, E., and Ali-Mehenni, M.: The rain profiling algorithm applied to polarimetric weather radar, J. Atmos. Ocean. Technol., 17, 332-356, 2000.

Vivekanandan, J., Zrnic, D. S., Ellis, S. M., Oye, R., Ryzhkov, A. V., and Straka, J.: Cloud microphysics retrieval using S-band dualpolarization radar measurements, B. Am. Meteorol. Soc.., 80, 381-388, 1999.

Vulpiani, G., Montopoli, M., Delli Passeri, L., Gioia, A. G., Giordano, P., and Marzano, F. S.: On the Use of Dual-Polarized CBand Radar for Operational Rainfall Retrieval in Mountainous Areas, J. Appl. Meteor. Climatol., 51, 405-425, 2012.

Wen, G., Protat, A., May, P. T., Wang, X., and Moran, W.: A Cluster-Based Method for Hydrometeor Classification Using Polarimetric Variables. Part I: Interpretation and Analysis, J. Atmos. Ocean. Technol., 32, 1320-1340, 2015.

Wen, G., Protat, A., May, P. T., Moran, W., and Dixon, M.: A Cluster-Based Method for Hydrometeor Classification Using Polarimetric Variables. Part II: Classification, J. Atmos. Ocean. Technol., 33, 45-60, 2016.

Witt, A., Eilts, M. D., Stumpf, G. J., Johnson, J. T., Mitchell, E. D., and Thomas, K. W.: An enhanced hail detection algorithm for the WSR-88D, Weather Forecast., 13, 286-303, 1998.

Wolfensberger, D., Scipion, D., and Berne, A.: Detection and characterization of the melting layer based on polarimetric radar scans, Q. J .Roy. Meteor. Soc., 142, 108-124, 2015.

Zrnic, D. S., Ryzhkov, A., Straka, J., Liu, Y., and Vivekanandan, J.: Testing a Procedure for Automatic Classification of Hydrometeor Types, J. Atmos. Ocean. Technol., 18, 892-913, 2001. 